\title{
NEUROPROSTHETIC BAROREFLEX CONTROLS HEMODYNAMICS AFTER SPINAL CORD INJURY
}

Jordan W. Squair ${ }^{1-6}$, Matthieu Gautier ${ }^{1 *}$, Lois Mahe ${ }^{1 *}$, Jan Elaine Soriano ${ }^{4 *}$, Andreas Rowald ${ }^{1 *}$, Arnaud Bichat ${ }^{1}$, Newton Cho ${ }^{1,9}$, Mark A. Anderson ${ }^{1}$, Nicholas D. James ${ }^{1}$, Jerome Gandar ${ }^{1}$, Anthony V. Incognito ${ }^{10}$, Giuseppe Schiavone ${ }^{11}$, Zoe K. Sarafis ${ }^{1}$, Achilleas Laskaratos ${ }^{1}$, Kay Bartholdi ${ }^{1}$, Robin Demesmaeker ${ }^{1,7}$, Salif Komii ${ }^{1,7}$, Charlotte Moerman7, Bita Vaseghi ${ }^{4}$, Berkeley Scott $^{4}$, Ryan Rosentreter ${ }^{4}$, Claudia Kathe ${ }^{1}$, Jimmy Ravier ${ }^{1}$, Laura McCracken ${ }^{1}$, Xiaoyang Kang ${ }^{11}$, Nicolas Vachicouras ${ }^{11}$, Florian Fallegger ${ }^{11}$, Ileana Jelescu ${ }^{12}$, YunLong Cheng ${ }^{13}$, Li Oin ${ }^{13}$, Rik Buschman ${ }^{14}$, Nicolas Buse ${ }^{14}$, Tim Denison ${ }^{15}$, Sean Dukelow ${ }^{4}$, Rebecca Charbonneau4, lan Rigby ${ }^{4}$, Steven K. Boyd ${ }^{16}$, Phillip J. Milar ${ }^{10}$, Eduardo Martin Moraud7, Marco Capogrosso ${ }^{8}$, Fabien B. Wagner $^{1,7}$, Quentin Barraud ${ }^{1}$, Erwan Bezard ${ }^{13,17,18}$, Stéphanie P. Lacour ${ }^{11}$, Jocelyne Bloch ${ }^{1,2,3,7}$, Grégoire Courtine ${ }^{1,2,3,7, \#}$ and Aaron A. Phillips ${ }^{4,6 \#}$

1. Center for Neuroprosthetics and Brain Mind Institute, School of Life Sciences, Swiss Federal Institute of Technology (EPFL), Lausanne, Switzerland

2. Department of Neurosurgery, Lausanne University Hospital (CHUV) and University of Lausanne (UNIL), Lausanne, Switzerland

3. Defitech Center for Interventional Neurotherapies, NeuroRestore, Switzerland

4. Department of Physiology and Pharmacology, Clinical Neurosciences, Cardiac Sciences, Hotchkiss Brain Institute, Libin Cardiovascular Institute of Alberta, Cumming School of Medicine, University of Calgary,

5. MD/PhD Training Program, Faculty of Medicine, University of British Columbia, Vancouver, Canada

6. International collaboration on repair discoveries (ICORD), University of British Columbia, Vancouver, Canada

7. Department of Clinical Neuroscience, Lausanne University Hospital (CHUV) and University of Lausanne (UNIL), Lausanne, Switzerland

8. Faculty of Biology, University of Fribourg, Fribourg, Switzerland

9. Department of Neurosurgery, University of Toronto, Toronto, Canada

10. Department of Human Health and Nutritional Sciences, University of Guelph, Guelph, Canada

11. Centre for Neuroprosthetics, Institute of Microengineering, Swiss Federal Institute of Technology (EPFL), Lausanne, Switzerland

12. Center for Biomedical Imaging, Swiss Federal Institute of Technology (EPFL), Lausanne, Switzerland

13. Motac Neuroscience Ltd, Manchester, United Kingdom

14. Medtronic, Minneapolis, USA

15. Oxford University, Oxford, England

16. Department of Radiology, McCaig Institute for Bone and Joint Health, University of Calgary

17. Université de Bordeaux, Institut des Maladies Neurodégénératives, UMR 5293, F-33000 Bordeaux, France

18. CNRS, Institut des Maladies Neurodégénératives, UMR 5293, F-33000 Bordeaux, France

*, \# contributed equally to this work

\section{Corresponding authors:}

Grégoire Courtine, PhD

gregoire.courtine@epfl.ch
Aaron A. Phillips, PhD

aaron.phillips@ucalgary.ca 
Spinal cord injury induces hemodynamic instability that threatens survival ${ }^{1-3}$, impairs neurological recovery ${ }^{4,5}$, increases cardiovascular disease risk $^{6,7}$, and reduces quality of life ${ }^{8,9}$. Hemodynamic instability in this context is due to the interruption of supraspinal excitatory drive to sympathetic circuits located in the spinal $\operatorname{cord}^{10}$, which impedes the natural baroreflex. Our previous studies showed that epidural electrical stimulation of the spinal cord transforms spinal circuits from a hypoactive to a highly active state ${ }^{11-16}$, and can reinstate spinal circuit dynamics to restore walking after paralysis ${ }^{17-19}$. Here, we leveraged these concepts to develop epidural electrical stimulation protocols that restored hemodynamic stability after spinal cord injury. We established a novel preclinical model that enabled us to dissect the topology and dynamics of the sympathetic circuits, and understand how epidural electrical stimulation can engage these circuits. We incorporated these spatial and temporal features into stimulation protocols to conceive a clinical-grade biomimetic hemodynamic regulator operating in closed-loop. This neuroprosthetic baroreflex controlled hemodynamics for extended periods of time in rodents, non-human primates, and humans, both after acute and chronic SCl. We will now conduct clinical trials to turn the neuroprosthetic baroreflex into a commonly available therapy for people with spinal cord injury.

Spinal cord injury $(\mathrm{SCl})$ immediately impairs hemodynamic stability, leading to repeated hypotensive episodes that are life threatening and reduce neurological recovery $1,4,5,20,21$. In the chronic phase, daily hypotensive episodes augment the risk of stroke and heart disease ${ }^{6,7}$, and reduce engagement in social and professional activities ${ }^{8,9}$.

Currently, the hemodynamic management of acute $\mathrm{SCl}$ is limited to long-acting pharmacological agents that are difficult to titrate and increase the incidence of adverse events ${ }^{3}$. Consequently, adherence to hemodynamic management guidelines remains poor ${ }^{22}$. Management strategies for chronic hypotension are limited and costly for unsatisfying efficacy ${ }^{23,24}$.

This hemodynamic instability is due to the interruption of supraspinal excitatory drive to the sympathetic circuitry, and thus disruption of the natural baroreflex. The resulting hypoactive state impairs the ability of the sympathetic circuitry to regulate hemodynamics ${ }^{25,26}$. Epidural electrical stimulation of the spinal cord can reactivate hypoactive circuits after $\mathrm{SCl}^{11,17-19}$. For example, epidural electrical stimulation targeting the lumbosacral posterior roots (TESS) increases the excitability of motor circuitry to a level that restores locomotion after $\mathrm{SCl}^{15,17,27}$. Serendipitous observations showed that epidural electrical stimulation of upper lumbar segments can also transiently elevate blood pressure ${ }^{28-31}$. However, the lumbosacral spinal cord contains a paucity of sympathetic efferent neurons, casting doubt that this approach harnesses the full potential of TESS to activate sympathetic circuits and achieve hemodynamic stability after SCl.

Here, we dissected the anatomical topology and physiological dynamics of the sympathetic circuitry, and uncovered the neural substrate through which TESS can modulate these circuits. We exploited this knowledge to conceive a biomimetic hemodynamic regulator operating in closed-loop that we implemented using clinical-grade implantable neurotechnologies ${ }^{17,27}$. This neuroprosthetic baroreflex precisely controlled hemodynamics over extended periods of time in 
rodents, non-human primates, and humans - effective from a few hours after $\mathrm{SCl}$ to the chronic phase of the condition.

\section{RESULTS}

\section{Preclinical model of hemodynamic instability}

Rats received a robotically-controlled, severe clinically-relevant contusion onto upper thoracic (T3) segments (Fig. 1a and Extended Data Fig. 1). To visualize how this contusion damaged the descending pathways regulating hemodynamics, we targeted catecholaminergic neurons expressing tyrosine hydroxylase $(\mathrm{TH})$ by stereotaxic infusions of AAV-DJ-hSyn-flex-mGFP-2ASynaptophysin-mRuby ${ }^{32}$ into the rostral ventrolateral medulla (RVLM) of TH-Cre rats ${ }^{33}$ (Fig. 1a). CLARITY-optimized light-sheet microscopy ${ }^{34}$ of $\mathrm{TH}^{\mathrm{ON}}$ fibers and synapses revealed a near complete depletion of sympatho-excitatory synapses onto ChAT ${ }^{\mathrm{N}}$ sympathetic preganglionic neurons below the injury (Fig. 1a).

To characterize the natural history of hemodynamic instability in response to $\mathrm{SCl}$, we implanted rats with a wireless system that enabled 24/7 monitoring of arterial blood pressure and sympathetic nerve activity (Fig. 1 b and Extended Data Fig. 1d-f). The SCl instantly induced a transient spike in blood pressure and sympathetic nerve activity (Extended Data Fig. 2b-c), followed by a pronounced depression that persisted throughout the chronic phase (Fig. 1d). 24/7 monitoring of blood pressure and sympathetic nerve activity in home cage revealed that the $\mathrm{SCl}$ led to profound hemodynamic instability (Extended Data Fig. 2d-g).

Despite the elimination of the natural baroreflex, the rats did not exhibit the hypotension observed in humans in response to orthostatic challenges (Extended Data Fig. 1a), thus preventing the investigation of this specific physiological mechanism in preclinical models.

To enable such investigations, we developed a servo-controlled negative pressure chamber that mimics orthostatic challenge paradigms used in humans to quantify hemodynamic instability (Fig. 1c). Uninjured rats exposed to negative pressure exhibited transient hypotensive episodes. After $\mathrm{SCl}$, rats could no longer recover from the simulated orthostatic challenge (Fig. 1d). They exhibited sustained hypotension, the severity of which linearly correlated with the pressure in the chamber (Extended Data Fig. 2i).

These results indicate that our preclinical model reproduced the hallmarks of hemodynamic instability observed in humans, and thus established heuristic conditions to dissect the mechanisms through which therapies could regulate hemodynamics after severe SCl.

\section{TESS engages the sympathetic circuitry to modulate hemodynamics}

We sought to reveal the optimal locations to target the sympathetic circuitry with TESS. We first identified the anatomical distribution of sympathetic pre-ganglionic neurons in the spinal cord using retrograde tracing from the splanchnic sympathetic ganglia, which can control blood pressure (Extended Data Fig. 3a). We found retrogradely-labelled neurons throughout the wellestablished sympathetic spinal cord topology 35 , but a peak concentration of neurons was 
identified in the low thoracic segments, centered around T12 (Fig. 2a and Extended Data Fig. 3b).

We then quantified the increase in blood pressure resulting from continuous TESS $(50 \mathrm{~Hz}$, motor threshold $)^{15}$ applied sequentially to each spinal segment, from T6 to L1. The pressor response to TESS followed a Gaussian distribution that also peaked around the low thoracic segments (Fig. 2a and Extended Data Fig. 3c-d). These responses were observed within a few hours after $\mathrm{SCl}$ and throughout the chronic phase (Extended Data Fig. 3d). Pressor responses to TESS linearly correlated with the density of retrogradely-labelled neurons, revealing a clear anatomical and functional enrichment at T11-T13 (Fig. 2a and Extended Data Fig. 3e). We named these segments the hemodynamic hotspots.

Next, we aimed to identify the circuit-level mechanisms through which TESS modulates hemodynamic hotspots; from the neural substrate recruited by TESS to the post-synaptic events that elicit constriction of blood vessels (Fig. $\mathbf{2 b}$ ).

We first modeled the electrical fields elicited by TESS using our previously-validated finite element methods ${ }^{36}$ that we complemented with high-resolution magnetic resonance imaging, computerized-tomography, and anatomical reconstructions of the low thoracic spinal cord (Fig. 2c). Simulations predicted that TESS applied to low thoracic segments primarily recruits largediameter afferent fibers $(A \alpha)$ located in the posterior roots, but has no direct influence on intraspinal neurons nor efferent pathways from sympathetic pre-ganglionic neurons (Extended

\section{Data Fig. 4a).}

To test this prediction, we asked whether the low thoracic posterior roots projecting to the hemodynamic hotspots were necessary to elicit pressor responses with TESS. We found that the progressive ablation of these roots led to the graded suppression of pressor responses (Fig. 2c and Extended Data Fig. 4b).

Previous clinical studies reported pressor responses when stimulating upper lumbar segments ${ }^{28-31}$, which appeared incongruent with our functional and anatomical mapping (Fig. 2a). We thus studied the mechanisms that could explain these observations. Computer simulations suggested that TESS applied at L2 can recruit the hemodynamic hotspot posterior roots where they bend and pass through the inter-vertebral foramen. Indeed, ablation of the T12 posterior roots blunted the modest pressor responses elicited by TESS applied at L2 (Extended Data Fig. 3f).

Computational modelling suggested that the recruitment of afferents with TESS engages sympathetic pre-ganglionic neurons through direct synaptic projections, excitatory interneurons, or both (Extended Data Fig. 4c). To explore these possibilities, we labelled afferent projections with co-injections of AAV-DJ-hSyn-flex-mGFP-2A-Synaptophysin-mRuby and AAV-Cre into T12 dorsal root ganglia. We developed a pipeline to dynamically warp spinal cord contours to a histological atlas, allowing us to merge data from multiple tissue sections and rats (Extended Data

Fig. 4d). Spatial analysis of merged matrices revealed an absence of afferent axons and synapses within the intermediolateral column that contains the vast majority of sympathetic pre-ganglionic neurons ${ }^{35}$ (Extended Data Fig. 4d). We confirmed the absence of the traced synaptic projections onto sympathetic pre-ganglionic neurons by immunolabelling them with choline acetyltransferase 
(ChAT). Therefore, excitatory interneurons likely mediated the activation of splanchnic sympathetic ganglion neurons. Indeed, time-dependent pseudorabies-mediated tracing revealed the presence of glutamatergic interneurons connected trans-synaptically to splanchnic sympathetic ganglionic neurons (Fig. 2d, Extended Data Fig. 4d). These interneurons were densely innervated by vGlut1 ${ }^{\text {ON }}$ synapses from proprioceptive afferents ${ }^{37}$ (Fig. 2e).

We next asked whether TESS recruits splanchnic sympathetic ganglion neurons, and whether their efferent pathways trigger pressor responses. Continuous TESS (30 $\mathrm{min}$ ) of hemodynamic hotspots induced a robust expression of the activity-dependent protein cFos in $\mathrm{TH}^{\mathrm{ON}}$ neurons located in splanchnic sympathetic ganglia (Fig. 2f). To ascertain their causal role, we expressed the light-sensitive eNpHR3.0 opsins ${ }^{38}$ in these neurons using targeted injections of AAV5-hSyn-eNpHR3.0-YFP in splanchnic sympathetic ganglia. Silencing of these neurons with light or ablating their efferent pathways blunted the pressor response to TESS (Fig. 2g, Extended Data Fig. 5c-d). These efferents release norepinephrine that induces constriction of blood vessels through the activation of alpha, receptors. We blocked these receptors with intravenous injections of prazosin 39 , which reversibly suppressed responses to TESS (Fig. 2h, Extended Data Fig. 5e).

These results show that TESS leads to alpha ${ }_{1}$ mediated constriction of blood vessels by recruiting proprioceptive afferent fibers that activate splanchnic neurons through a circuitry involving glutamatergic interneurons directly connected to sympathetic preganglionic neurons.

\section{Closed-loop control of hemodynamics through biomimetic stimulation}

We exploited this knowledge to configure and fabricate electronic dura mater (e-dura) implants ${ }^{40}$ that targeted the posterior roots projecting to the hemodynamic hotspots. Optimal electrode numbers and locations were identified using a genetic algorithm ${ }^{15,17}$ implemented in the computational model of TESS. We optimized the geometry and thickness of e-dura implants to conform to the curved topology of the low thoracic spinal column (Fig. 3a and Extended Data Fig. 6a-c).

Neuromodulation strategies that mimic natural dynamics are more effective than unspecific protocols ${ }^{15,17,41}$. Moreover, non-physiological manipulation of hemodynamics may damage the central nervous system and circulatory system while predisposing to adverse events ${ }^{42}$. We therefore aimed to deliver TESS patterns that mimic the natural dynamics of sympathetic circuitry activation. To capture these dynamics, we recorded the activity of neurons located in the RVLM in conjunction with sympathetic nerve activity and hemodynamics (Extended Data Fig. 6de). Feed-forward, artificial neural networks confirmed the disrupted link between RVLM activity and sympathetic circuitry after $\mathrm{SCl}$ (Extended Data Fig. 6f). Wavelet decomposition analysis ${ }^{43}$ of blood pressure signals revealed that blue-light photostimulation of $\mathrm{ChR} 2^{\mathrm{ON}} \mathrm{TH}^{\mathrm{ON}}$ neurons located in the RVLM steers hemodynamics within specific frequency domains ${ }^{44}$ confined around 0.4-1.0 $\mathrm{Hz}$ (Fig. 3b). Orthostatic challenges enhanced hemodynamic activity within the same frequency band in uninjured rats (Fig. 3c). This drive was permanently interrupted after severe SCl (Fig. 3c).

We then measured the rostrocaudal transmission of descending sympathetic volleys (Extended Data Fig. 6g), which we quantified from the propagation of surface potentials over the 
hemodynamic hotspots in response to stimulation of RVLM neurons. We found a conduction delay of 2.5 ms between activation of adjacent hotspots.

We encoded these biometrics into TESS protocols that consequently mimic the spatial sequences, frequency contents and temporal profiles underlying natural sympathetic circuit activation. This biomimetic stimulation reinstated the natural dynamics of the system (Fig. 3d, Extended Data Fig. 6h), and triggered greater pressor responses than conventional TESS protocols (Fig. 3a).

The management of hemodynamic instability logically necessitates constant titration of TESS. We found that biomimetic stimulation led to pressor responses that linearly correlated with TESS amplitude $\left(R^{2}=0.81 ; P=1.02 e^{-15}\right)$. Therefore, we implemented a proportional-integral $(\mathrm{PI})$ controller that modulated biomimetic TESS protocols to target user-defined blood pressure levels in closed-loop ${ }^{15}$ (Fig. 4a). This hemodynamic regulator updates TESS amplitudes in real-time to prevent hypotension (Fig. 4b-c). We thus conceived a neuroprosthetic baroreflex that rapidly (1.15s 95\% Cl: 0.36-2.5) stabilized hemodynamics during transient, varying, and sustained orthostatic challenges, both after acute and chronic SCl (Fig. 4b-e and Extended Data Fig. 7ce). To directly compare this neuroprosthetic baroreflex to the natural baroreflex, we conceived a brain-spine interface ${ }^{45}$ that established a proportional link between the cumulative firing of RVLM neurons and biomimetic stimulation protocols (Extended Data Fig. 7g). This link effectively stabilized hemodynamics following orthostatic challenges, with the same temporal delay as the natural baroreflex measured in uninjured rats (Extended Data Fig. 2). The neuroprosthetic baroreflex operated tenfold faster (Extended Data Fig. 7h-i).

\section{Translational implementation of the neuroprosthetic baroreflex}

We next asked whether this neuroprosthetic baroreflex could stabilize hemodynamics immediately after SCl using clinical-grade neurotechnologies (Extended Data Fig. 8c) ${ }^{17,27}$. We emulated neuro-intensive care in a non-human primate model that included general propofolbased anesthesia, an arterial pressure line, and artificial ventilation (Fig. 5a-b, Extended Data Fig. 8a-b).

We mapped pressor responses to TESS across the thoracic and lumbar spinal cords of three Rhesus monkeys with acute complete upper-thoracic (T3) SCl, which confirmed the location of hemodynamic hotspots within the three most caudal thoracic segments (Extended Data Fig. 9a-b).

To modulate these hemodynamic hotspots, we designed e-dura implants ${ }^{40}$ with electrode configurations that targeted the left and right posterior roots of T10, T11, and T12 segments. We scaled the implants developed for rats to the anatomical features of Rhesus monkeys, measured in three animals (Extended Data Fig. 9c). We connected these e-dura implants to a clinical-grade implantable pulse generator with wireless communication modules and custom-made software interfaces that enable real-time control over spatial locations, temporal sequences, and amplitudes of TESS ${ }^{17}$. We injected all the features of the neuroprosthetic baroreflex within this versatile stimulation platform (Extended Data Fig. 8c). 
The complete $\mathrm{SCl}$ induced an immediate spike in blood pressure, rapidly followed by pronounced hypotension reminiscent of life-threatening hemodynamic instability observed acutely in humans with $\mathrm{SCl}$ (Extended Data Fig. 8d-e).

As early as a few hours after the complete $\mathrm{SCl}$, the neuroprosthetic baroreflex instantly normalized blood pressure, stabilizing hemodynamics for extensive periods of time without the need for supervision (2'000 heartbeats shown in Fig. 5c). Contrary to closed-loop TESS, pressor responses induced by continuous TESS extinguished after a few heartbeats in this acute phase of $\mathrm{SCl}$ (Extended Data Fig. 9g-h). The neuroprosthetic baroreflex maintained hemodynamic stability despite pronounced orthostatic challenges induced in a negative pressure chamber adapted to Rhesus monkeys (Fig. 5d and Extended Data Fig. 9f,i). During hemodynamic collapse, the neuroprosthetic baroreflex completely rescued hemodynamic stability (Fig. 5e and

\section{Supplementary Video 1).}

\section{Clinical implementation of hemodynamic stabilization}

We finally aimed to validate our therapeutic framework to stabilize hemodynamics in humans. To enable future clinical trials, we focused on validating the key features of the neuroprosthetic baroreflex in a patient presenting with a chronic functionally complete cervical $\mathrm{SCl}$ that led to debilitating, medically-refractory orthostatic hypotension (Extended Data Fig. 10a).

We first confirmed that the hemodynamic hotspots are also located in the low thoracic spinal cord in humans. A paddle electrode array was surgically positioned below the T10 and T11 vertebral bodies that contain the posterior roots entering lower thoracic segments (Extended Data Fig. 10a). Computer simulations predicted the optimal electrode configurations ${ }^{17}$ to target the hemodynamic hotspots identified in preclinical models (Fig. 6a). TESS induced robust pressor responses (Fig. 6b), whereas stimulation delivered more caudally or over electrodes non-specific for the posterior roots (midline) was comparatively far less effective (Extended Data Fig. 10c-d). TESS increased sympathetic nerve activity and normalized circulating levels of norepinephrine, which confirmed the activation of the sympathetic circuitry (Fig. 6c).

We then asked whether tuning the amplitude of TESS could regulate hemodynamics in closed-loop. As observed in preclinical models, we found a linear relationship between stimulation amplitudes and pressor responses (Extended Data Fig. 10e). Graded adjustment of TESS amplitude led to real-time hemodynamic stabilization during orthostatic challenges on a tilttable (Fig. 6d and Extended Data Fig. 10f-g).

Long-term implementation of TESS enabled the permanent cessation of medical treatments for hemodynamic stabilization, increased participation in verticalized motor rehabilitation, and abolished the clinical burden of orthostatic hypotension (Extended Data Fig.

\section{0h and Supplementary Video 2 ).}

\section{DISCUSSION}

We developed and validated an ultrafast, highly-reliable neuroprosthetic baroreflex that precisely stabilizes hemodynamics in the acute and chronic phases of SCl. Central to this development was 
a novel heuristic preclinical model of hemodynamic instability that enabled the identification of the topology and dynamics of natural sympathetic circuit activation. We combined this fundamental knowledge with a new understanding of the mechanisms through which TESS activates the sympathetic circuitry to conceive biomimetic TESS protocols that obey guidelines for ecoprosthetic designs ${ }^{46}$. The evolutionary conservation of the ancestral sympathetic circuitry enabled straightforward translation of these protocols from rats to non-human primates to humans.

We implemented this neuroprosthetic baroreflex within an implantable stimulation platform that we previously used to deliver biomimetic TESS protocols controlled in closed-loop to restore walking after paralysis in humans with $\mathrm{SCl}^{17}$. We validated this neuroprosthetic baroreflex in non-human primates, and experiments in one patient with chronic tetraplegia indicate that the human spinal cord responds effectively to the key features of this treatment. Therefore, this clinical-grade investigational device will now enable clinical trials to evaluate the safety and therapeutic efficacy of the neuroprosthetic baroreflex in the acute, sub-acute and chronic phases of $\mathrm{SCl}$.

In parallel, it is imperative to develop a fully implantable system combining a paddle electrode array targeting the hemodynamic hotspots, an arterial blood pressure monitoring unit, and a closed-loop stimulation platform that embodies all the necessary features to deliver biomimetic TESS protocols for hemodynamic stabilization. The neuroprosthetic baroreflex foreshadows a new era in the clinical management of $\mathrm{SCl}$. 


\section{REFERENCES}

1. Squair, J. W., Phillips, A. A., Harmon, M. \& Krassioukov, A. V. Emergency management of autonomic dysreflexia with neurologic complications. Can. Med. Assoc. J. 188, 1100-1103 (2016).

2. Readdy, W. J. et al. Complications and outcomes of vasopressor usage in acute traumatic central cord syndrome. J Neurosurg Spine 23, 574-580 (2015).

3. Inoue, T., Manley, G. T., Patel, N. \& Whetstone, W. D. Medical and surgical management after spinal cord injury: vasopressor usage, early surgerys, and complications. J. Neurotrauma 31, 284-291 (2014).

4. Squair, J. W. et al. Spinal cord perfusion pressure predicts neurologic recovery in acute spinal cord injury. Neurology 89, 1660-1667 (2017).

5. Squair, J. W. et al. Empirical targets for acute hemodynamic management of individuals with spinal cord injury. Neurology 93, e1205-e1211 (2019).

6. Cragg, J. J., Noonan, V. K., Krassioukov, A. \& Borisoff, J. Cardiovascular disease and spinal cord injury: results from a national population health survey. Neurology 81, 723-728 (2013).

7. Wu, J.-C. et al. Increased risk of stroke after spinal cord injury: a nationwide 4-year follow-up cohort study. Neurology 78, 1051-1057 (2012).

8. Illman, A., Stiller, K. \& Williams, M. The prevalence of orthostatic hypotension during physiotherapy treatment in patients with an acute spinal cord injury. Spinal Cord 38, 741-747 (2000).

9. Carlozzi, N. E. et al. Impact of blood pressure dysregulation on health-related quality of life in persons with spinal cord injury: development of a conceptual model. Arch. Phys. Med. Rehabil. 94, 1721-1730 (2013).

10. Furlan, J. C., Fehlings, M. G., Shannon, P., Norenberg, M. D. \& Krassioukov, A. V. Descending vasomotor pathways in humans: correlation between axonal preservation and cardiovascular dysfunction after spinal cord injury. J. Neurotrauma 20, 1351-1363 (2003).

11. Courtine, G. et al. Transformation of nonfunctional spinal circuits into functional states after the loss of brain input. Nat. Neurosci. 12, 1333-1342 (2009).

12. Musienko, P., van den Brand, R., Maerzendorfer, O., Larmagnac, A. \& Courtine, G. Combinatory electrical and pharmacological neuroprosthetic interfaces to regain motor function after spinal cord injury. IEEE Trans. Biomed. Eng. 56, 2707-2711 (2009).

13. Asboth, L. et al. Cortico-reticulo-spinal circuit reorganization enables functional recovery after severe spinal cord contusion. Nat. Neurosci. 21, 576-588 (2018).

14. van den Brand, R. et al. Restoring voluntary control of locomotion after paralyzing spinal cord injury. Science 336, 1182-1185 (2012).

15. Wenger, N. et al. Spatiotemporal neuromodulation therapies engaging muscle synergies improve motor control after spinal cord injury. Nat. Med. 22, 138-145 (2016). 
16. Takeoka, A., Vollenweider, I., Courtine, G. \& Arber, S. Muscle spindle feedback directs locomotor recovery and circuit reorganization after spinal cord injury. Cell 159, 1626-1639 (2014).

17. Wagner, F. B. et al. Targeted neurotechnology restores walking in humans with spinal cord injury. Nature 563, 65-71 (2018).

18. Angeli, C. A. et al. Recovery of Over-Ground Walking after Chronic Motor Complete Spinal Cord Injury. N. Engl. J. Med. 379, 1244-1250 (2018).

19. Gill, M. L. et al. Neuromodulation of lumbosacral spinal networks enables independent stepping after complete paraplegia. Nat. Med. 24, 1677-1682 (2018).

20. Saadoun, S., Chen, S. \& Papadopoulos, M. C. Intraspinal pressure and spinal cord perfusion pressure predict neurological outcome after traumatic spinal cord injury. J. Neurol. Neurosurg. Psychiatry jnnp-2016-314600 (2016).

21. Vale, F. L., Burns, J., Jackson, A. B. \& Hadley, M. N. Combined medical and surgical treatment after acute spinal cord injury: results of a prospective pilot study to assess the merits of aggressive medical resuscitation and blood pressure management. $J$. Neurosurg. 87, 239-246 (1997).

22. Kong, C. Y. et al. A prospective evaluation of hemodynamic management in acute spinal cord injury patients. Spinal Cord 51, 466-471 (2013).

23. Krassioukov, A., Eng, J. J., Warburton, D. E., Teasell, R. \& Spinal Cord Injury Rehabilitation Evidence Research Team. A systematic review of the management of orthostatic hypotension after spinal cord injury. Arch. Phys. Med. Rehabil. 90, 876885 (2009).

24. Phillips, A. A. \& Krassioukov, A. V. Contemporary Cardiovascular Concerns after Spinal Cord Injury: Mechanisms, Maladaptations, and Management. J. Neurotrauma 32, 1927-1942 (2015).

25. Phillips, A. A., Krassioukov, A. V., Ainslie, P. N. \& Warburton, D. E. R. Baroreflex function after spinal cord injury. J. Neurotrauma 29, 2431-2445 (2012).

26. Phillips, A. A., Krassioukov, A. V., Ainslie, P. N. \& Warburton, D. E. R. Perturbed and spontaneous regional cerebral blood flow responses to changes in blood pressure after high-level spinal cord injury: the effect of midodrine. J. Appl. Physiol. 116, 645653 (2014).

27. Capogrosso, M. et al. A brain-spine interface alleviating gait deficits after spinal cord injury in primates. Nature 539, 284-288 (2016).

28. West, C. R. et al. Association of epidural stimulation with cardiovascular function in an individual with spinal cord injury. JAMA Neurol. 75, 630-632 (2018).

29. Harkema, S. J. et al. Epidural spinal cord stimulation training and sustained recovery of cardiovascular function in individuals with chronic cervical spinal cord injury. JAMA Neurol. 75, 1569-1571 (2018).

30. Harkema, S. J. et al. Normalization of blood pressure with spinal cord epidural stimulation after severe spinal cord injury. Front. Hum. Neurosci. 12, 83 (2018).

31. Darrow, D. et al. Epidural Spinal Cord Stimulation Facilitates Immediate Restoration of Dormant Motor and Autonomic Supraspinal Pathways after Chronic 
Neurologically Complete Spinal Cord Injury. J. Neurotrauma 36, 2325-2336 (2019).

32. Grimm, D. et al. In vitro and in vivo gene therapy vector evolution via multispecies interbreeding and retargeting of adeno-associated viruses. J. Virol. 82, 5887-5911 (2008).

33. Witten, I. B. et al. Recombinase-driver rat lines: tools, techniques, and optogenetic application to dopamine-mediated reinforcement. Neuron 72, 721-733 (2011).

34. Tomer, R., Ye, L., Hsueh, B. \& Deisseroth, K. Advanced CLARITY for rapid and highresolution imaging of intact tissues. Nat. Protoc. 9, 1682-1697 (2014).

35. Strack, A. M., Sawyer, W. B., Marubio, L. M. \& Loewy, A. D. Spinal origin of sympathetic preganglionic neurons in the rat. Brain Res. 455, 187-191 (1988).

36. Capogrosso, M. et al. A computational model for epidural electrical stimulation of spinal sensorimotor circuits. J. Neurosci. 33, 19326-19340 (2013).

37. Beauparlant, J. et al. Undirected compensatory plasticity contributes to neuronal dysfunction after severe spinal cord injury. Brain 136, 3347-3361 (2013).

38. Gradinaru, V., Thompson, K. R. \& Deisseroth, K. eNpHR: a Natronomonas halorhodopsin enhanced for optogenetic applications. Brain Cell Biol 36, 129-139 (2008).

39. Phillips, A. A., Elliott, S. L., Zheng, M. M. Z. \& Krassioukov, A. V. Selective alpha adrenergic antagonist reduces severity of transient hypertension during sexual stimulation after spinal cord injury. J. Neurotrauma 32, 392-396 (2015).

40. Minev, I. R. et al. Biomaterials. Electronic dura mater for long-term multimodal neural interfaces. Science 347, 159-163 (2015).

41. Formento, E. et al. Electrical spinal cord stimulation must preserve proprioception to enable locomotion in humans with spinal cord injury. Nat. Neurosci. 21, 1728-1741 (2018).

42. Cornwell, W. K. et al. Restoration of Pulsatile Flow Reduces Sympathetic Nerve Activity Among Individuals With Continuous-Flow Left Ventricular Assist Devices. Circulation 132, 2316-2322 (2015).

43. Saleem, S. et al. Wavelet decomposition analysis is a clinically relevant strategy to evaluate cerebrovascular buffering of blood pressure after spinal cord injury. Am. J. Physiol. Heart Circ. Physiol. 314, H1108-H1114 (2018).

44. Phillips, A. A., Warburton, D. E. R., Ainslie, P. N. \& Krassioukov, A. V. Regional neurovascular coupling and cognitive performance in those with low blood pressure secondary to high-level spinal cord injury: improved by alpha-1 agonist midodrine hydrochloride. J. Cereb. Blood Flow Metab. 34, 794-801 (2014).

45. Bonizzato, M. et al. Brain-controlled modulation of spinal circuits improves recovery from spinal cord injury. Nat. Commun. 9, 3015 (2018).

46. Courtine, G. \& Bloch, J. Defining ecological strategies in neuroprosthetics. Neuron 86, 29-33 (2015). 
Supplementary Information is linked to the online version of the paper at www.nature.com/nature.

Acknowledgements. The NRP and investigational implantable stimulators were donated from Medtronic PLC. This work was supported by the Wings for Life Foundation (Project Grant), Compute Canada (Resources for Research Groups), the Natural Sciences and Engineering Research Council (Canada; Discovery Grant), the Canadian Institutes of Health Research (Project Grant; Banting Fellowship J.W.S), Alberta Innovates Health Solutions (Postdoctoral Fellowship J.W.S), Campus Alberta Neuroscience, the Libin Cardiovascular Institute of Alberta, the Hotchkiss Brain Institute, the Rick Hansen Institute, International Foundation for Research in Paraplegia (IRP), McCaig Institute for Bone and Joint Health, European Union's Horizon 2020 Framework Programme for Research and Innovation under the Specific Grant Agreement No. 720270 (Human Brain Project SGA1) No. 785907 (Human Brain Project SGA2), and No. 896791 (Marie SkłodowskaCurie Individual Fellowship J.W.S.) the Marie Skłodowska-Curie grant program No. 665667, RESTORE: Eurostars E10889, OPTISTIM: Eurostars E!12743, WALKAGAIN: Eurostars E!11834, the Swiss National Science Foundation including the National Center of Competence in Robotics and subside 310030_185214, and Sinergia subside CRSII3_160696, a Consolidator Grant from the European Research Council [ERC-2015-CoG HOW2WALKAGAIN 682999], and the Bertarelli Foundation. Prof. Luc Stoppini and his team (HEPIA, Geneva) for their help with ETO sterilization. We thank Sim4Life by ZMT, www.zurichmedtech.com for their support. We thank Brian K. Kwon for critically reading the manuscript and his insightful suggestions.

\section{Author Contributions.}

M.G., J.E.S., Lo.M., and A.R. contributed equally to this work. G.C. and A.A.P. contributed equally to this work. J.W.S., M.G., Lo.M., J.E.S., J.B., G.C., and A.A.P. carried out the experiments. J.W.S., M.G., Lo.M., J.E.S., L. Mc., C.M., and E.M.M. analyzed the data. A.R. and Ma.C. designed and implemented the computational framework. A.A.P, J.W.S, and G.C conceived of the closed-loop lower body negative pressure chamber. A.R. conducted computational simulations. J.W.S, J.E.S, A.B., M.A.A., N.D.J. performed the rodent surgeries. J.B., G.C., E.B., Y.C., and L.Q. conducted and oversaw the non-human primate experiments. N.C. performed all tissue clearing procedures. J.W.S., N.C., and Q.B. coordinated light-sheet imaging. J.W.S., M.A.A., and K.B. performed viral tract tracing. J.W.S. and N.D.J. performed all electrophysiology studies. J.W.S., J.G., A.A.P, I.R., and E.M.M. conceived all stimulation-based closed-loop protocols. J.W.S., J.G., and E.M.M. implemented rodent closed-loop protocols. R.D., S.K., C.M., F.B.W., and E.M.M. implemented non-human primate closed-loop protocols. A.I. and P.M. developed approaches for human muscle sympathetic nerve recordings. A.l. carried out muscle sympathetic nerve recordings. J.W.S., M.G., Lo.M., Z.K.S., A.L., K.B., C.K., and Q.B. carried out all anatomical analyses. G.S., X.K., N.V., F.F., and S.P.L. designed, fabricated, and characterised the soft electrode arrays. B.V. and A.A.P. prepared human ethics protocols. B.V., B.S., R.R., R.C, E.S., S.D, S.B, A.I., and A.A.P collected human participant data. S.B., A.A.P, and BV facilitated CT and MRI scanning for human participant. 
I.J. carried out MRI-sequences in rodents. S.D. conducted clinical examinations. R.B., N.B. and T.D. led the development of the Neural Research Programmer and the associated investigational implantable stimulator. J.W.S., M.G., Lo.M., J.E.S., A.R., Q.B., and J.R. generated the figures. J.R. conceived the illustrations. G.C. and A.A.P. conceived and supervised the study. G.C. and A.A.P. secured funding. G.C., A.A.P. and J.W.S. wrote the paper, and all the authors contributed to its editing.

Author Information. Data that supports the findings and software routines developed for the data analysis will be made available upon reasonable request to the corresponding authors at gregoire.courtine@epfl.ch or aaron.phillips@ucalgary.ca. Reprints and permissions information is available at www.nature.com/reprints. The authors declare competing financial interests: G.C., J.B., J.W.S., A.A.P. and S.P.L hold various patents in relation with the present work. T.D., R.B. and N.B are Medtronic employees. Y.C. and L.Q. are Motac neuroscience employees. E.B. is consultant and shareholder of Motac neuroscience. In review of the manuscript they contributed to technical accuracy but did not influence the results or the content of the manuscript. G.C., J.B. and S.P.L are founders and shareholders of GTXmedical, a company with potential relationships with the presented invention. 


\section{FIGURE 1}

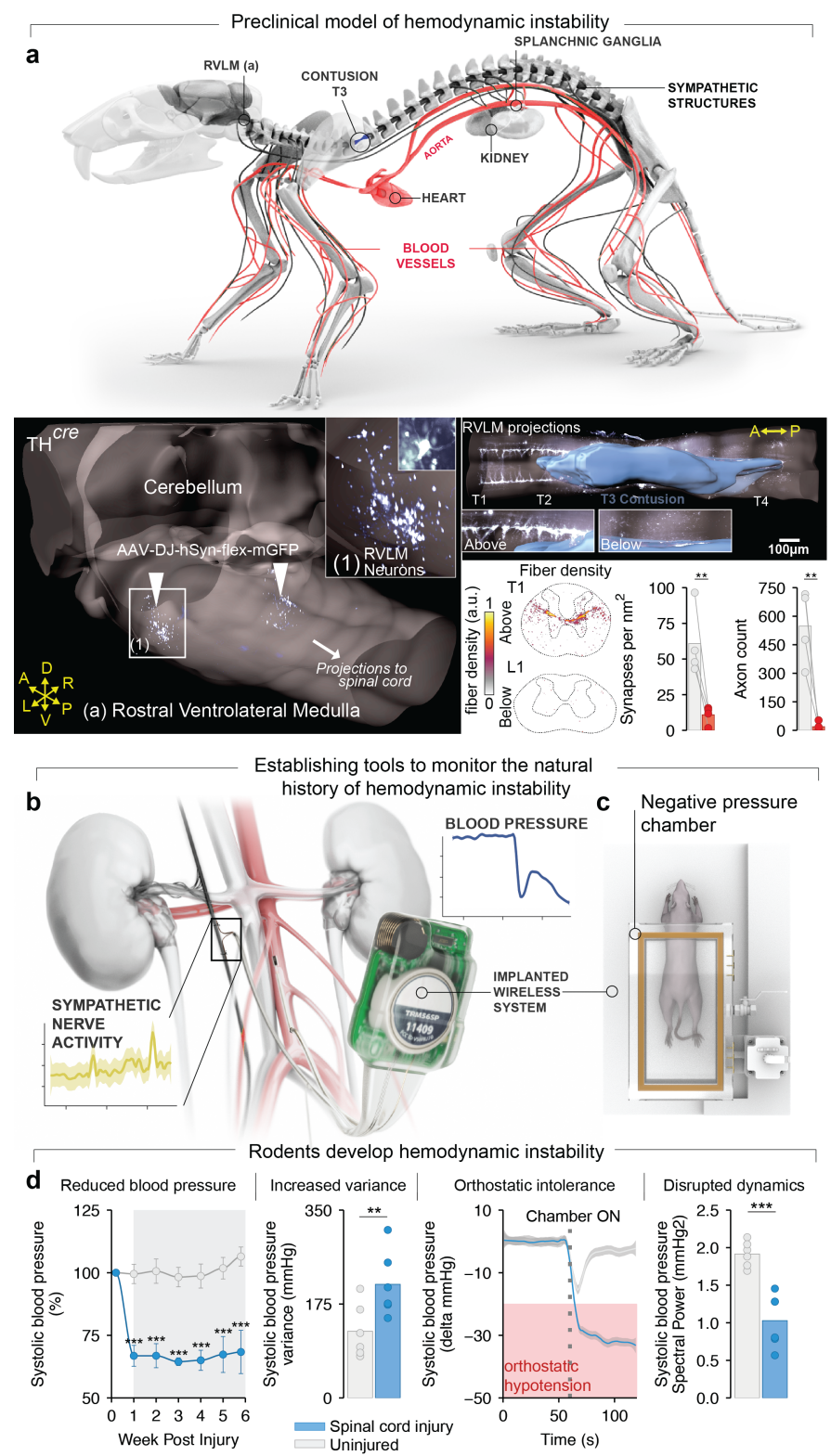

\section{Fig. 1 | Preclinical model overview.}

a, Sympathetic nervous system underlying hemodynamic control. $\mathrm{TH}^{\mathrm{ON}}$ neurons in the RVLM and their descending projections targeted with injections of AAV-DJ-hSyn-flex-mGFP-2A-Synaptophysin-mRuby in $\mathrm{TH}:$ Cre rats, imaged in the brainstem (3D rendering, uDISCO) and contused spinal cord (light-sheet microscopy, CLARITY). Density of descending sympatho-excitatory tract fibers are shown above and below the contusion. Barplots report mean fiber density $(n=4)$ (paired one-tailed t-test; $t=5.08 ; P=0.007$ ) and synapses (paired one-tailed $\mathrm{t}$-test; $\mathrm{t}=4.64 ; P=0.009$ ).

b, Rats implanted with wireless telemetry system to record hemodynamics and sympathetic nerve activity 24/7.

c, Negative pressure chamber inducing blood pressure reductions, similar to a clinical 'tilt-test'.

d, Monitoring of resting blood pressure before and for 6 weeks post-injury ( $n=6$, two-way repeated measures ANOVA followed by Tukey's honestly significant difference (HSD) post-hoc correction; $F_{(1,6) i n t e r a c t i o n}$ $=7.05 ; P=1.10 \mathrm{e}^{-05}$; all post-hoc $<0.001$ ). Barplots report hemodynamic variance (independent samples one-tailed t-test; $\mathrm{t}=2.80 ; P=0.009$ ) and low frequency pressure dynamics (independent samples one-tailed $\mathrm{t}$-test; $\mathrm{t}=5.51 ; P=0.0004)$. The line plot illustrates orthostatic intolerance after injury and when exposed to negative pressure. ${ }^{\star} P<0.05 ;{ }^{\star \star} P<0.01 ;{ }^{\star \star \star} P<0.001$. 


\section{FIGURE 2}

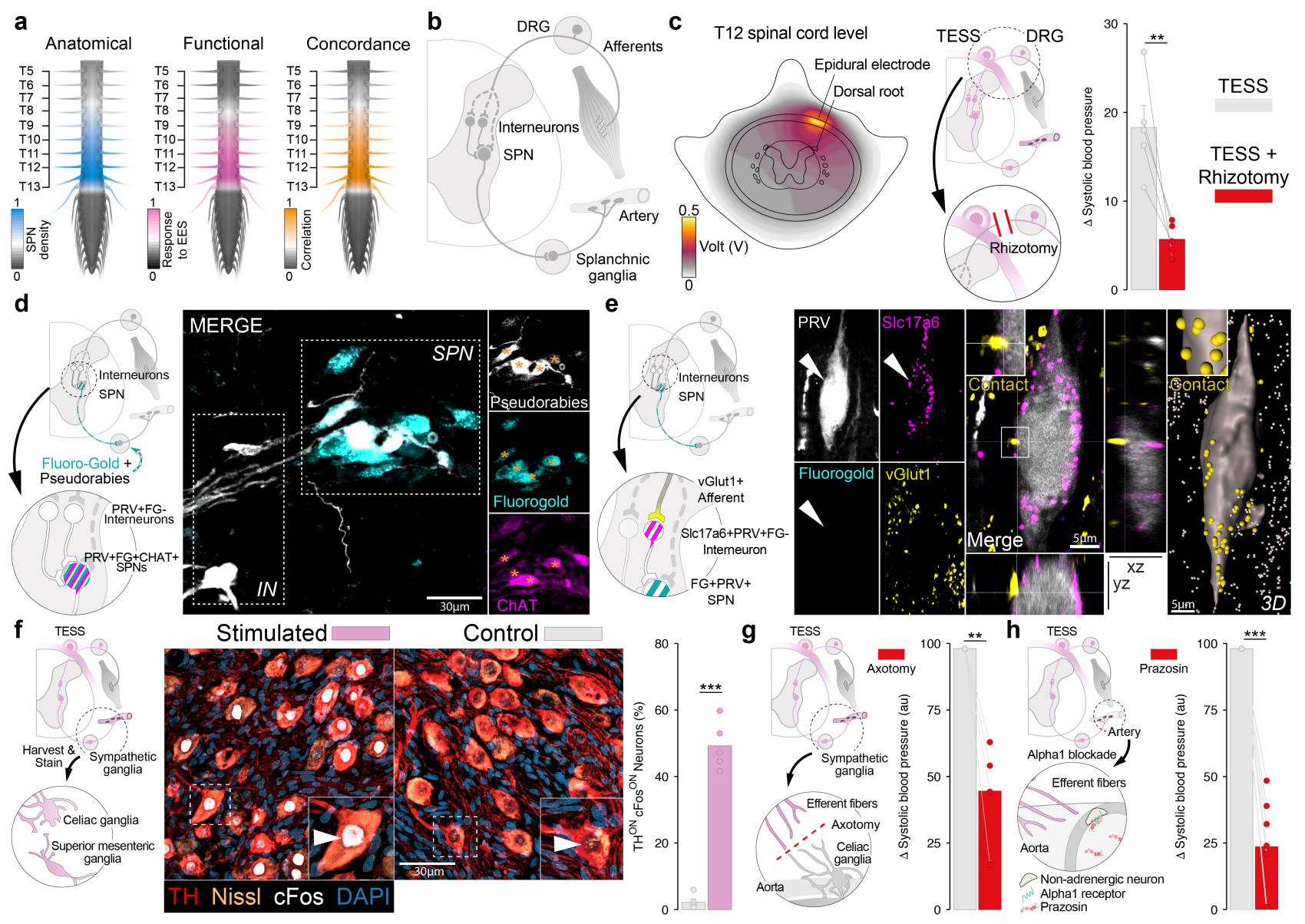

Fig. 2 | Mechanisms by which EES stabilizes hemodynamics.

a, Intraspinal density of neurons retrogradely traced from the splanchnic ganglia, amplitude of pressor responses to TESS applied to each segment, and concordance between anatomical and functional datasets.

b, Hypothetical circuits activated by TESS to elicit blood vessel constriction.

c, Color-coded electrical potentials following TESS applied to the spinal cord suggesting the exclusive activation of afferent fibres. Scheme illustrating rhizotomy of posterior roots. Barplots report pressor responses to TESS before and after rhizotomy $(n=5$, paired samples one-tailed t-test; $t=4.36 ; P=0.006)$.

d, Trans-synaptic retrograde tracing revealing interneurons connected to splanchnic ganglia.

e, These interneurons express the excitatory marker Slc17a6, and receive vGlut1 synapses from largediameter proprioceptive afferents.

f, Fos expression in $\mathrm{TH}^{\mathrm{ON}}$ neurons in the splanchnic ganglia in control and after TESS. Barplot reports percentage of $\mathrm{FOS}^{\mathrm{ON}}$ neurons ( $n=5$, independent samples one-tailed $t$-test; $t=13.96 ; P=4.99 \mathrm{e}^{-05}$ ).

g, Ablation of splanchnic efferents blunted the pressor response $(n=4$, independent samples one-tailed $t$ test; $\mathrm{t}=-4.54 ; P=0.0099$ ).

h, alpha1 receptor blockade with prazosin blunted pressor responses ( $n=5$, independent samples onetailed t-test; $\mathrm{t}=-5.59 ; P=0.0007$ ). 
FIGURE 3

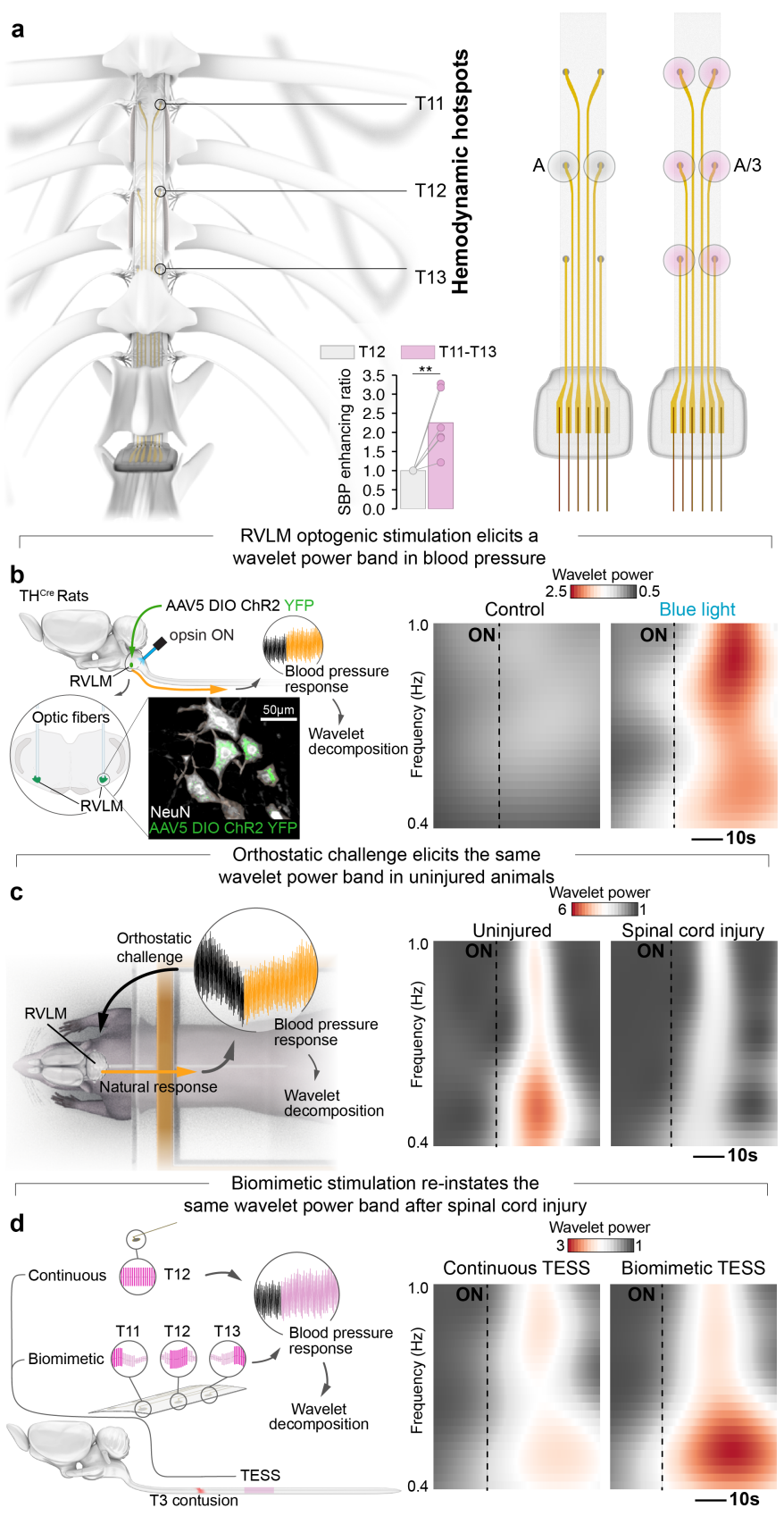

Fig. 3 | Biomimetic stimulation protocols to control hemodynamics.

a, Schematic of electronic dura mater electrode arrays targeting hemodynamic hotspots. Barplot reports relative pressor responses (SBP: systolic blood pressure; $\mathrm{n}=5$, independent samples one-tailed $\mathrm{t}$-test; $\mathrm{t}=$ 3.90; $P=0.006$ ).

b, Targeted expression of ChR2 in TH ${ }^{\mathrm{ON}}$ neurons of the RVLM, as shown in the photograph. Wavelet power spectrum when illuminating the RVLM with yellow (control) versus blue light (paired samples one-tailed ttest; $\mathrm{t}=2.44 ; P=0.035$ ).

c, Wavelet spectrogram when inducing an orthostatic challenge by decreasing the pressure in the chamber $(\mathrm{n}=6$; independent samples one-tailed $\mathrm{t}$-test; $\mathrm{t}=3.01 ; P=0.0013$ ).

d, Schematic illustrating biomimetic stimulation protocols, composed of interleaved $(2.5 \mathrm{~ms}) \mathrm{TESS}(5 \mathrm{OHz})$ propagating over the hemodynamic hotspots. Wavelet spectrogram in rats with $\mathrm{SCl}(\mathrm{n}=5$, paired samples one-tailed t-test; $\mathrm{t}=2.31 ; P=0.041$ ). 


\section{FIGURE 4}
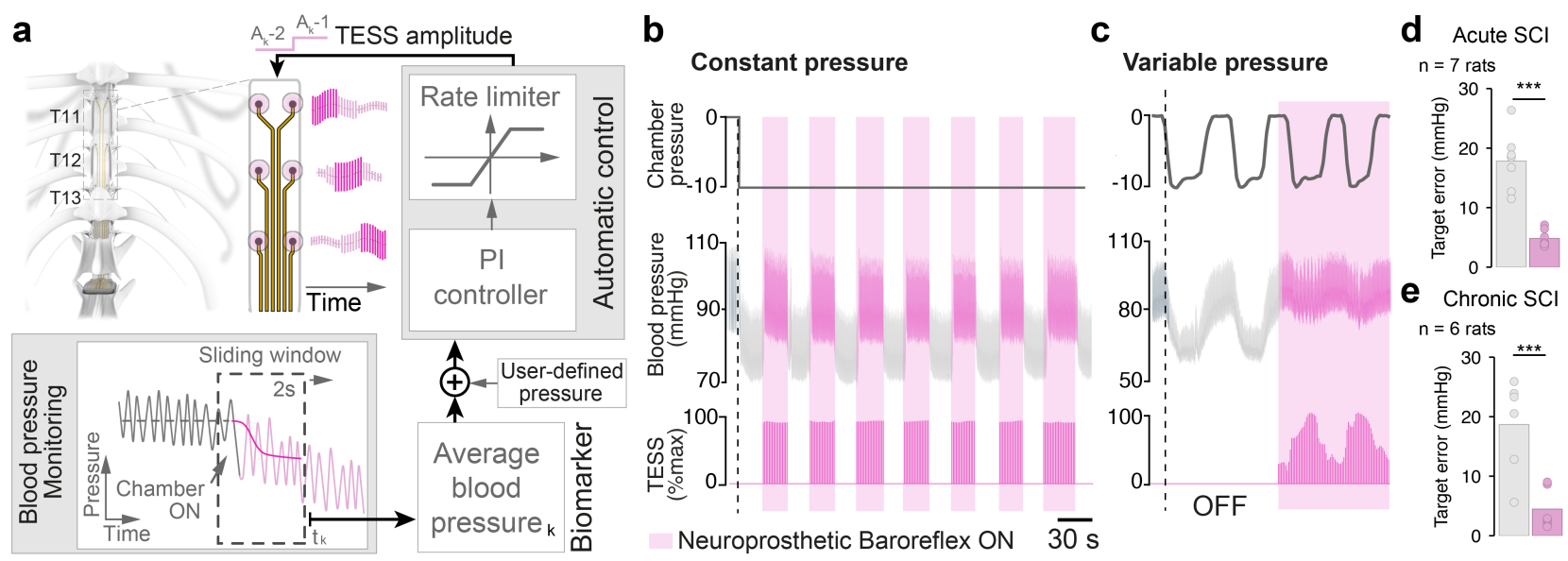

Fig. 4 | Neuroprosthetic baroreflex implemented in rats with severe contusion.

a, Closed-loop controller of blood pressure using a proportional integral (PI) controller that adjust the amplitude of traveling TESS waves over the three cardiovascular hotspots to maintain a user-defined blood pressure (target).

b, Pressure in the chamber, blood pressure and TESS amplitude while the neuroprosthetic baroreflex is turned on and off sequentially.

c, Same variables as in $\mathbf{b}$ shown for cyclical changes in the pressure of the chamber. Barplot reporting errors in blood pressure with respect to the user-defined target with the neuroprosthetic baroreflex on and off, tested in rats with

d, acute (1-3 hours post-injury, $n=7$, paired samples one-tailed t-tests; systolic blood pressure (SBP): $t=$ $\left.6.12, P=5.50 \mathrm{e}^{-04}\right)$ and

e, chronic ( 6 weeks, $n=6$, paired samples one-tailed t-tests; SBP: $\mathrm{t}=-3.84, P=0.006$ ) $\mathrm{SCl}$ (see Extended Data Figure 7). 


\section{FIGURE 5}

a

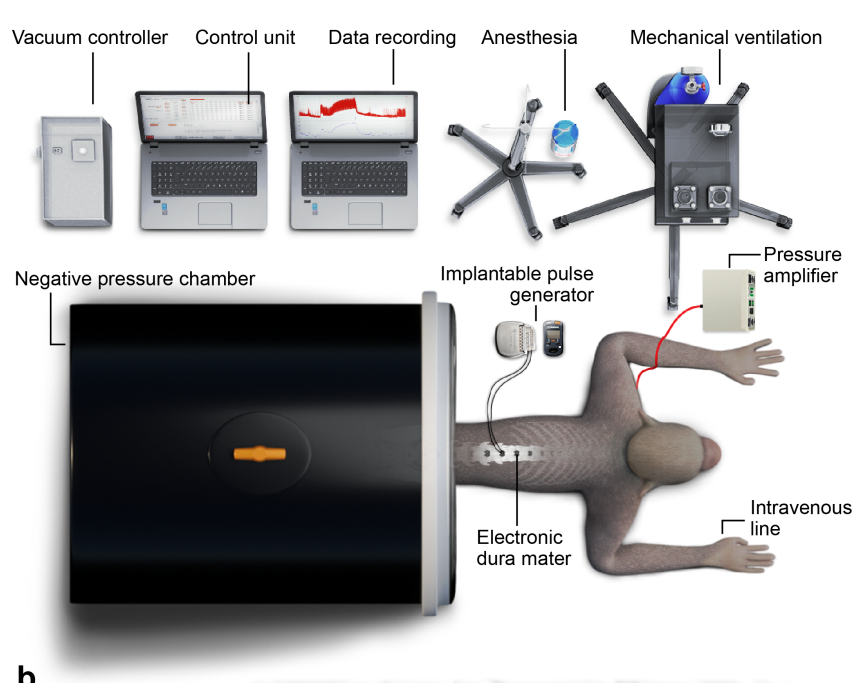

C

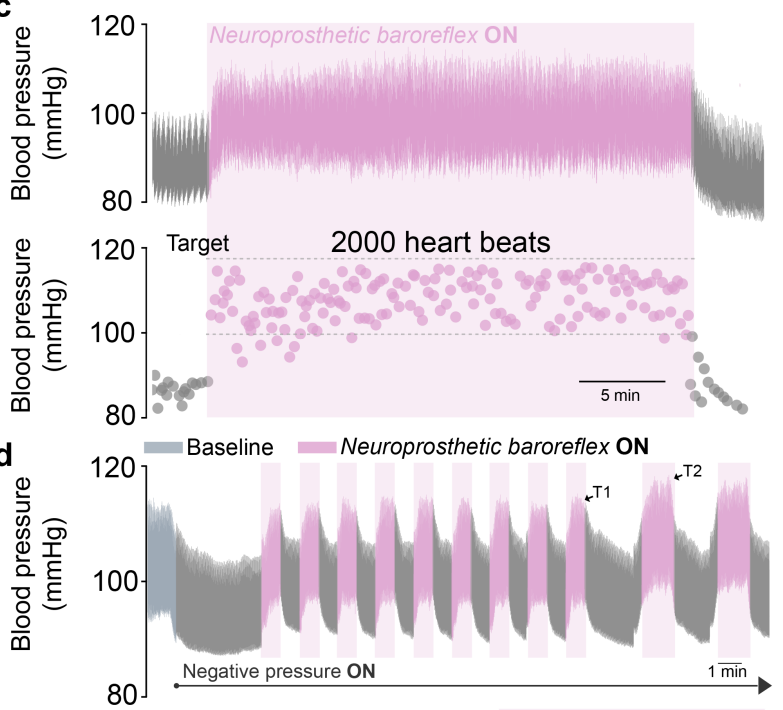

e

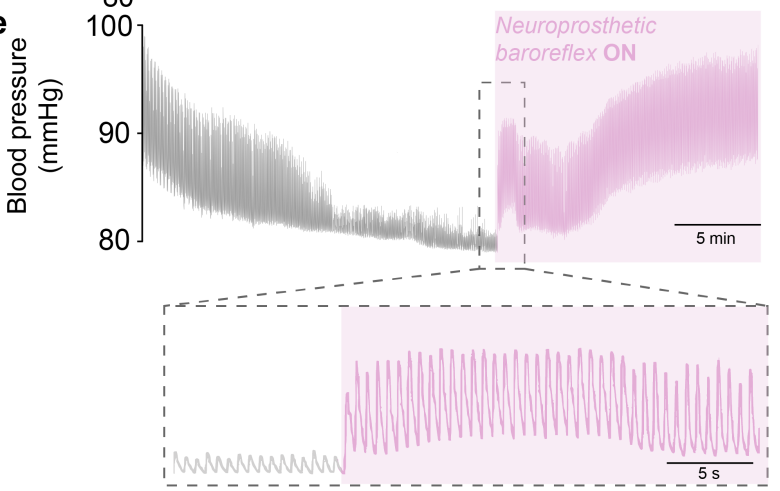

Fig. 5 | Neuroprosthetic baroreflex implemented in non-human primates with complete SCI.

a, Neurointensive care unit for rhesus monkeys with acute complete upper thoracic $\mathrm{SCl}$, placed in a negative pressure chamber. The neuroprosthetic baroreflex is implemented using clinical-grade technologies operated with a custom-made software.

b, Localization of electronic dura matter.

c, Blood pressure with the neuroprosthetic baroreflex on for 2000 heartbeats.

d, Same experiments as in Figure 4b. T1 = first target, $\mathrm{T} 2$ = second target.

e, Blood pressure collapse, rescued by the neuroprosthetic baroreflex. 


\section{FIGURE 6}
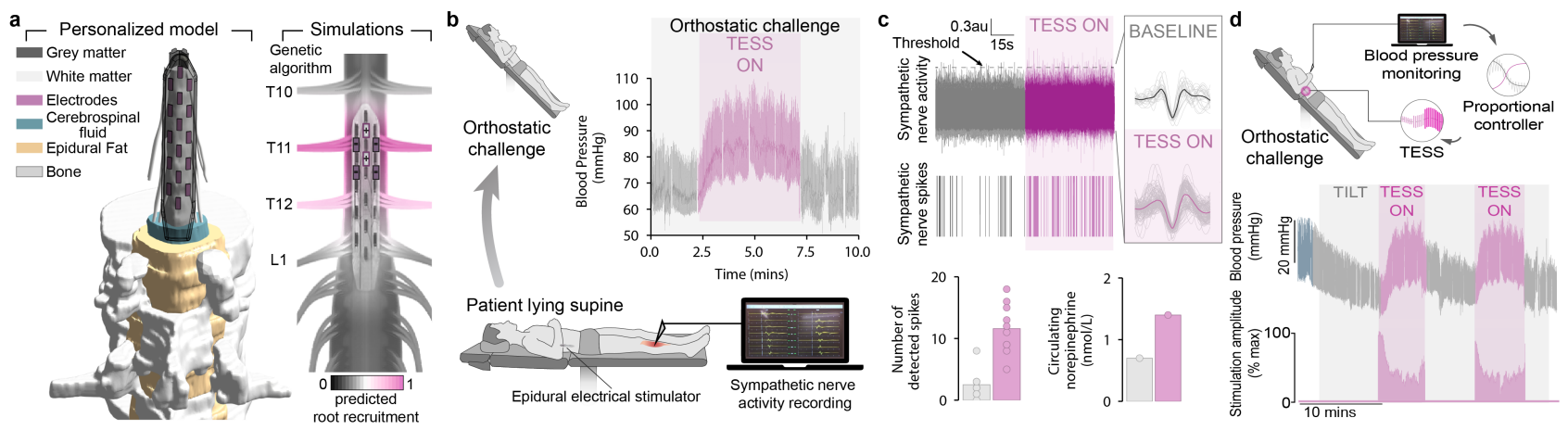

Fig. 6 | Key features of the neuroprosthetic baroreflex implemented in an individual with a functionally complete $\mathbf{C 5} \mathbf{S C l}$.

a, Personalized computational model of the spine, spinal cord and implanted stimulating lead. A genetic algorithm predicted the optimal spatial configurations (+, anode; -, cathode) to activate the cardiovascular hotspots identified in rodents and non-human primates.

b, Orthostatic challenge on a tilt table. Pressor response with TESS applied with the configuration predicted by the model.

c, Sympathetic nerve recording without and with TESS. Barplots report spike counts and levels of circulating norepinephrine without and with TESS.

d, Pressor responses during closed-loop control of TESS amplitude concomitant to orthostatic challenges on a tilt-table. 


\section{EXTENDED DATA FIGURE 1}

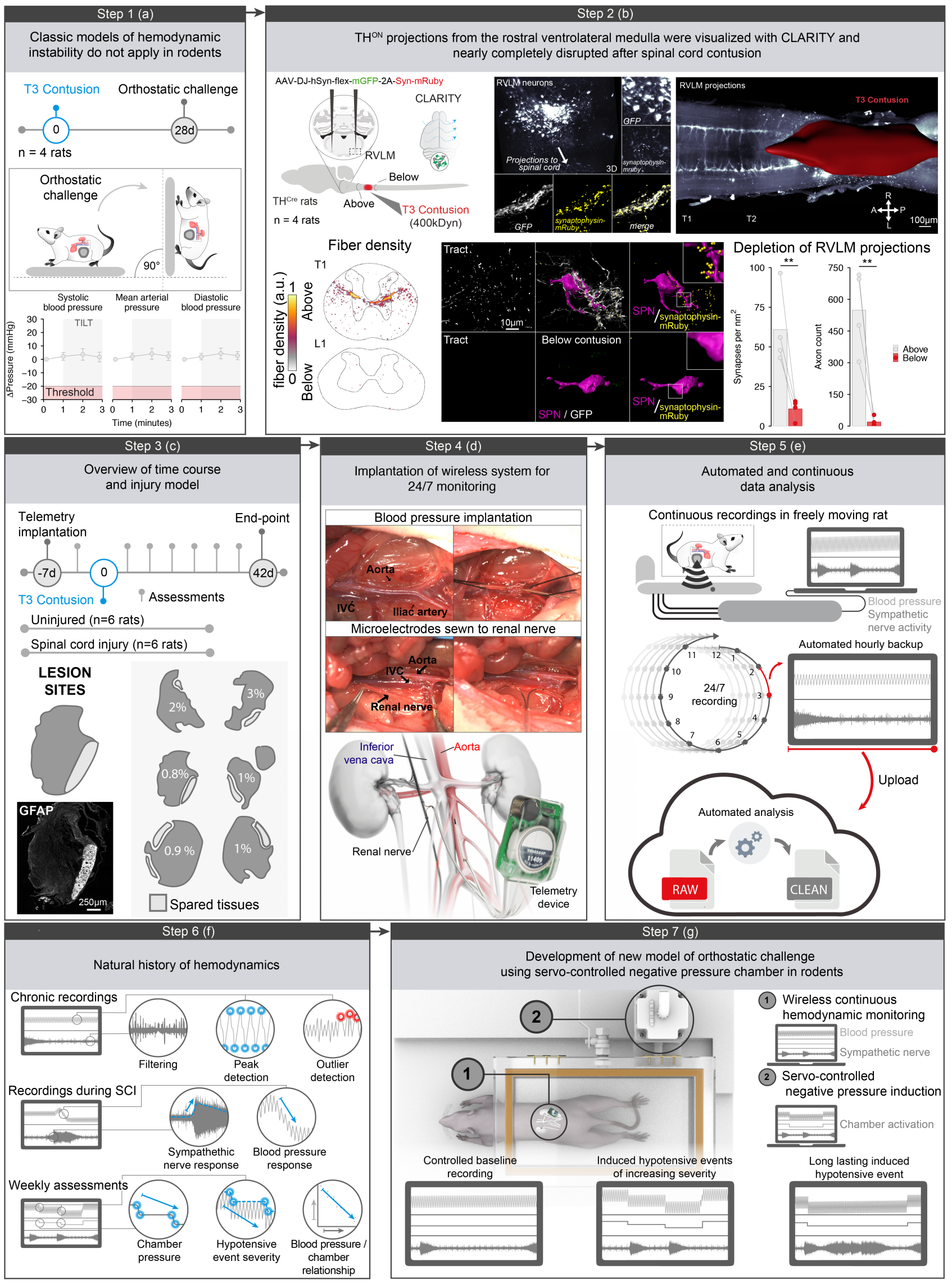




\section{Extended Data Figure 1. Development of a novel model of hemodynamic instability in rodents.}

Step 1(a): We first tested the capacity for an orthostatic challenge to reduce blood pressure in rats 30 days after a T3 spinal cord injury $(n=4)$. Tilting rats 90 degrees upright did not lead to any reduction in systolic blood pressure (one-way repeated measures ANOVA; $F_{3}=0.612 ; P=0.62$ ), diastolic blood pressure (oneway repeated measures $A N O V A ; F_{3}=1.105 ; P=0.40$ ), or mean arterial pressure (one-way repeated measures ANOVA; $\left.F_{3}=0.915 ; P=0.47\right)$.

Step 2(b): To confirm that our contusion model disrupted descending control of hemodynamics we used AAV-DJ-hSyn-flex-mGFP-2A-Synaptophysin-mRuby injected into the rostral ventrolateral medulla (RVLM) of tyrosine hydroxylase $(\mathrm{TH})$-Cre rats $(n=4)$ one month after T3 spinal cord injury. We found a near complete disruption of descending $\mathrm{TH}^{\mathrm{ON}}$ sympatho-excitatory axons (paired one-tailed t-test; $\mathrm{t}=5.08 ; P=0.007$ ) and synapses (paired one-tailed t-test; $\mathrm{t}=4.64 ; P=0.009$ ) when comparing counts above and below the injury. Barplots represent the mean with raw data overlaid.

Step 3(c): Overview of the time-course used to examine the natural history of hemodynamic instability in uninjured $(n=6)$ and spinal cord injured animals $(n=6)$. Confirmation that the lesion site spared minimal white matter (identified using glial fibrillary acidic protein [GFAP]; mean $=2 \%$ ).

Step 4(d): We implanted a wireless recording system to monitor hemodynamics and sympathetic nerve activity. A blood pressure cannula was inserted into the abdominal aorta and microelectrodes sutured to the sympathetic renal nerve.

Step 5(e): Data was recorded 24/7 and automatically uploaded to a server where automated analyses were triggered to quantify blood pressure and sympathetic nerve activity throughout the day and night.

Step 6(f): We established the natural history of hemodynamic instability by recording the response to spinal cord injury and automatically detecting outliers (see Supplementary Online Methods, Hemodynamic and sympathetic nerve activity monitoring) for blood pressure and sympathetic nerve activity data for a total of 7 weeks.

Step 7(g): Because rats do not exhibit hemodynamic instability in response to an orthostatic challenge (see Step 1(a)), we developed a servo-controlled negative pressure approach whereby animals are placed in a chamber and the pressure is dropped and monitored in closed loop (see Supplementary Online Methods, Implementation of an orthostatic challenge in rodents and non-human primates).

${ }^{\star} P<0.05 ;{ }^{\star \star} P<0.01 ;{ }^{\star \star \star} P<0.001$. 


\section{EXTENDED DATA FIGURE 2}

a

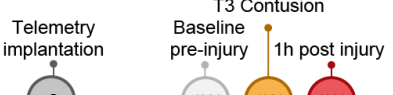

- 10 (iiv)-(iiv) (iii) -

Chronic catheterization $y=r$
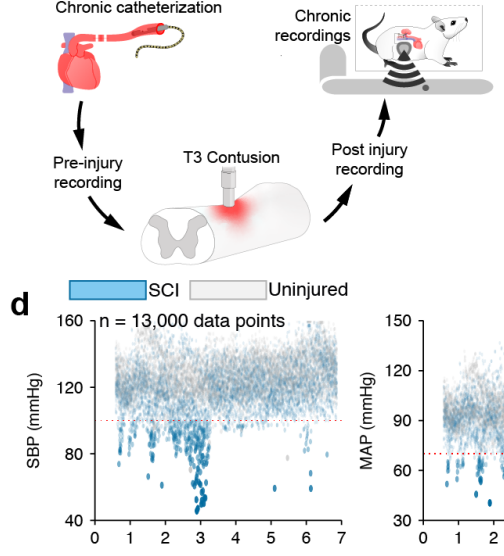

b

BASELINE CONTUSION

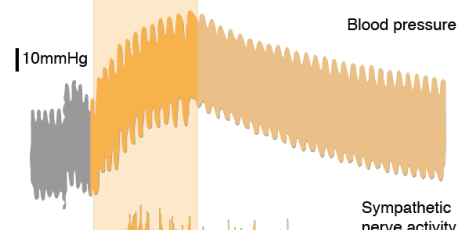

pouv
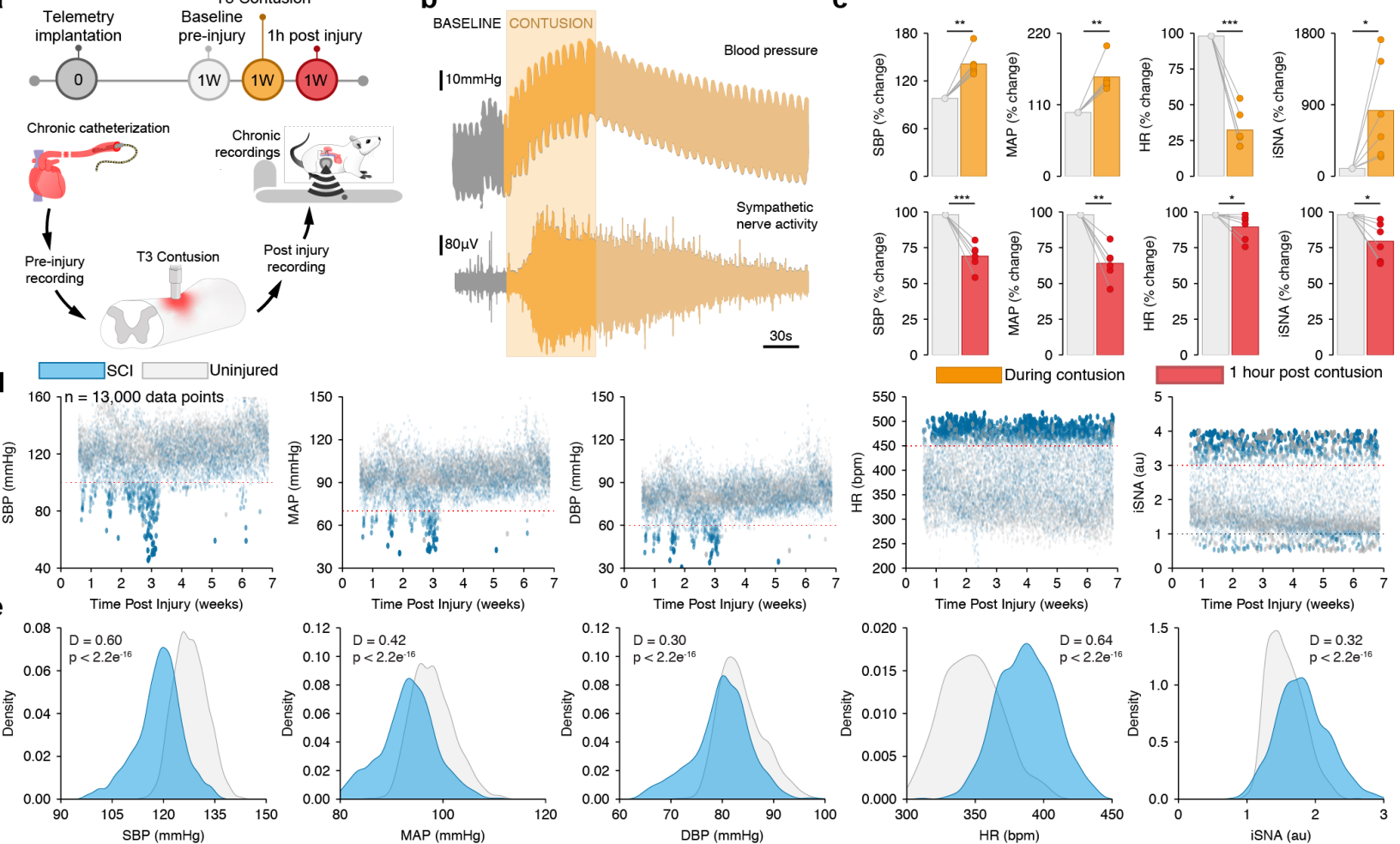
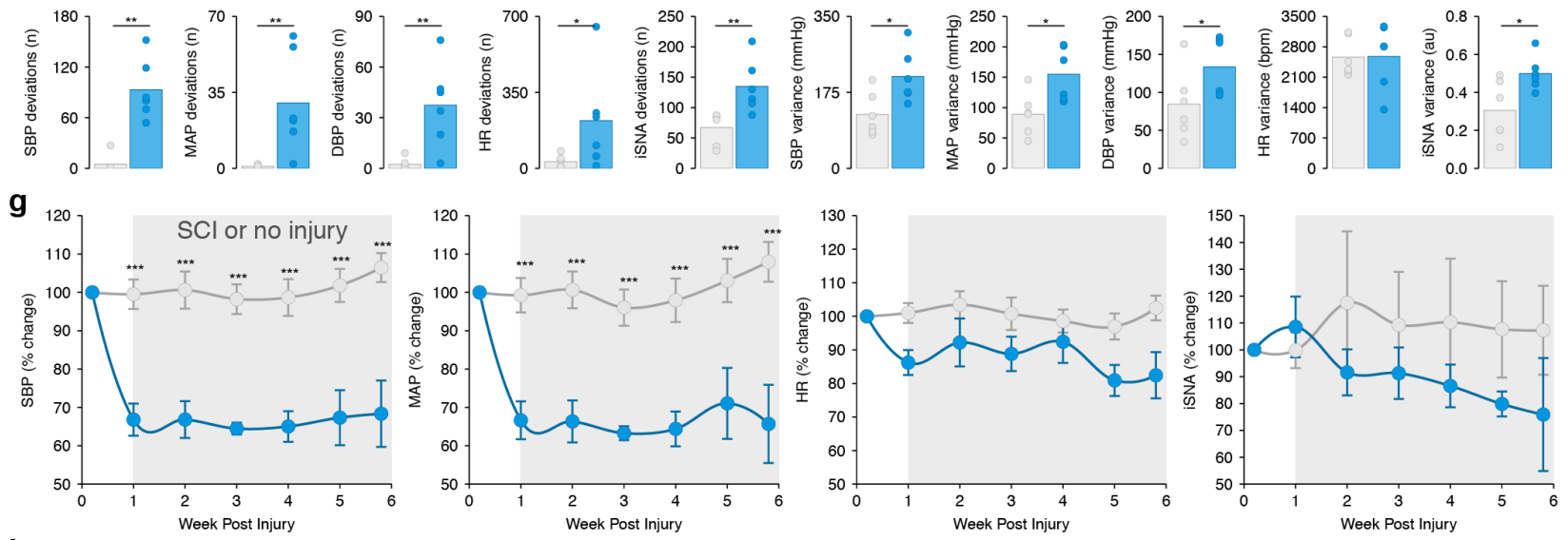

h
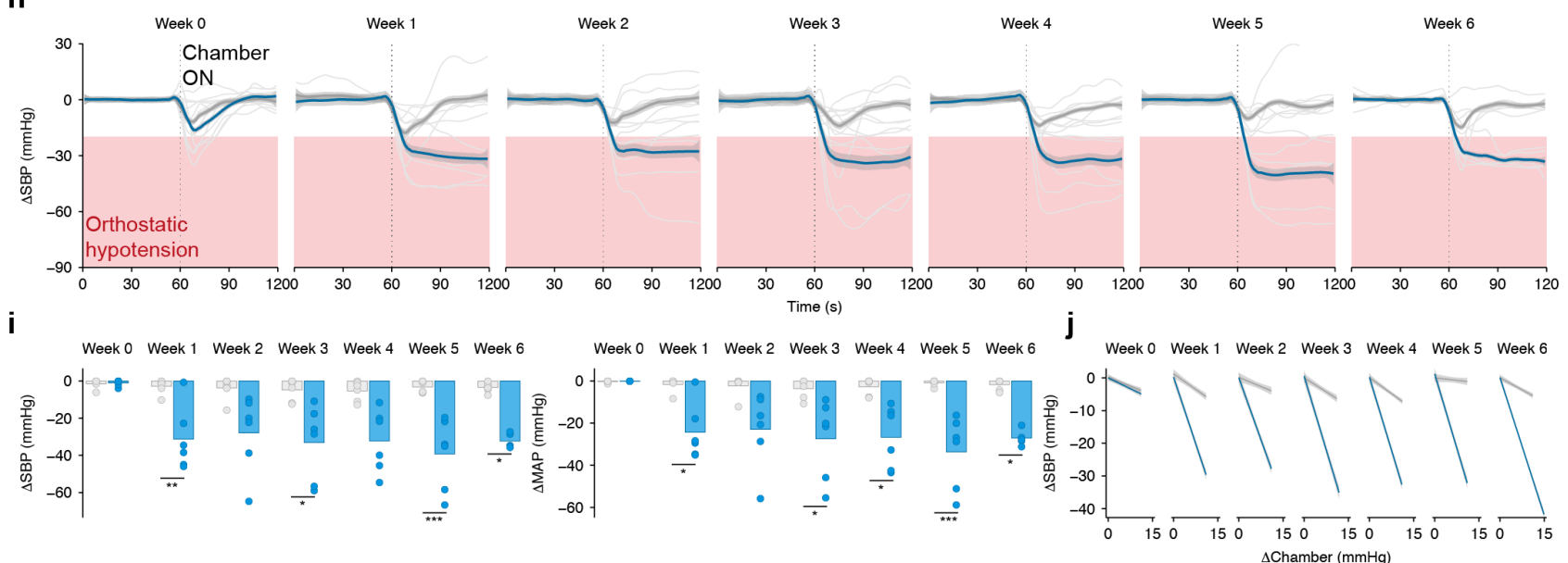


\section{Extended Data Figure 2. Rodent model develops hemodynamic instability across the natural history of spinal cord injury.}

a, We recorded hemodynamics and sympathetic nerve activity during and after the spinal cord contusion $(n=6)$.

b, We observed an immediate increase in blood pressure and sympathetic nerve activity following the onset of the contusion.

c, Quantifications revealed a significant increase in systolic blood pressure (paired one-tailed t-test; $t=5.40$; $P=0.001$ ) and mean arterial pressure (paired one-tailed t-test; $t=4.50 ; P=0.003$ ), a decrease in heart rate (paired one-tailed t-test; $\mathrm{t}=-10.26 ; P=7.56 \mathrm{e}^{-05}$ ), and an increase in sympathetic nerve activity (paired onetailed $\mathrm{t}$-test; $\mathrm{t}=2.26 ; P=0.037$ ) during contusion. These were followed by complete reversal after the contusion, where systolic blood pressure (paired one-tailed t-test; $t=-5.97 ; P=0.0009$ ), mean arterial pressure (paired one-tailed t-test; $\mathrm{t}=-5.14 ; P=0.002$ ), sympathetic nerve activity (paired one-tailed $\mathrm{t}$-test; $\mathrm{t}$ $=-3.29 ; P=0.011$ ), and heart rate (paired one-tailed t-test; $\mathrm{t}=-2.91 ; P=0.017$ ) decreased compared to pre-injury.

d, We next established the natural history of hemodynamics using 24/7 recordings. We found that throughout the recording period animals with spinal cord injury deviated outside key thresholds representing 'normal' values (points scaled by size and transparency based on their deviation outside our set thresholds [dotted lines]).

e, We found a left-shift in the distribution of hemodynamic values, and a right-ward shift in heart rate values, indicating generally lower blood pressure, higher heart rate, and more aberrant sympathetic nerve activity (Kolmogorov-Smirnov test; all $\mathrm{P}<2.2 \mathrm{e}^{-16}$ ).

f, Quantifications revealed an increase in the number of deviations for systolic blood pressure (independent samples one-tailed t-test; $\mathrm{t}=5.92 ; P=0.0005$ ), diastolic blood pressure (independent samples one-tailed $\mathrm{t}$-test; $\mathrm{t}=3.68 ; P=0.007$ ), mean arterial pressure (independent samples one-tailed $\mathrm{t}$-test; $\mathrm{t}=3.23 ; P=$ 0.011 ), heart rate (independent samples one-tailed t-test; $\mathrm{t}=2.0 ; P=0.0499$ ), and sympathetic nerve activity (independent samples one-tailed t-test; $\mathrm{t}=3.20 ; P=0.006$ ). We additionally found an increase in the variance of systolic blood pressure (independent samples one-tailed t-test; $\mathrm{t}=2.70 ; P=0.011$ ), diastolic blood pressure (independent samples one-tailed t-test; $\mathrm{t}=2.01 ; P=0.036$ ), mean arterial pressure (independent samples one-tailed t-test; $\mathrm{t}=2.85 ; P=0.009$ ), and sympathetic nerve activity (independent samples one-tailed t-test; $\mathrm{t}=3.20 ; P=0.006$ ), fitting the criteria for hemodynamic instability after spinal cord injury.

9, Formal baseline recordings revealed that baseline systolic blood pressure (two-way repeated measures ANOVA; interaction effect $\mathrm{F}_{6,1}=7.05 ; P=1.10 \mathrm{e}^{-05}$; all Tukey post-hoc $\mathrm{P}<0.001$ ) and mean arterial pressure (two-way repeated measures ANOVA; interaction effect $F_{6,1}=4.93 ; P=0.0004$; all Tukey post-hoc $P<0.001$ ) were reduced, in agreement with spontaneous $24 / 7$ data recordings.

$\mathbf{h}$, We devised a closed-loop negative pressure system to mimic an orthostatic challenge in rats. Animals with spinal cord injury could not respond to decreasing pressures, whereas uninjured animals responded and slowly recovered. This response was consistent across all six weeks post injury and is contrasted against week 0 (no injury for both groups).

i, Quantification of this response revealed increased negative deltas for the spinal cord injury group for systolic blood pressure (two-way repeated measures ANOVA; interaction effect $F_{6,1}=3.80 ; P=0.003$; all Tukey post-hoc $P<0.05$ ) and mean arterial pressure (two-way repeated measures ANOVA; interaction effect $\mathrm{F}_{6,1}=4.12 ; P=0.001$; all Tukey post-hoc $\left.P<0.05\right)$.

j, After $\mathrm{SCl}$ we observed a time-dependent increase in the linear relationship between chamber pressure and blood pressure (likelihood ratio test of nested models; $\mathrm{P}<0.001$ ), indicating that hemodynamics cannot be stabilized during orthostatic challenge without a functioning baroreflex. In all panels, percent change is presented for clarity as needed, while all statistics are calculated from raw values. Bar plots represent the mean with raw data overlaid.

${ }^{\star} P<0.05 ;{ }^{* \star} P<0.01 ;{ }^{* \star *} P<0.001$.

Abbreviations: iSNA: integrated sympathetic nerve activity; HR: heart rate; MAP: mean arterial pressure; SBP: systolic blood pressure; SCI: spinal cord injury. 


\section{EXTENDED DATA FIGURE 3}

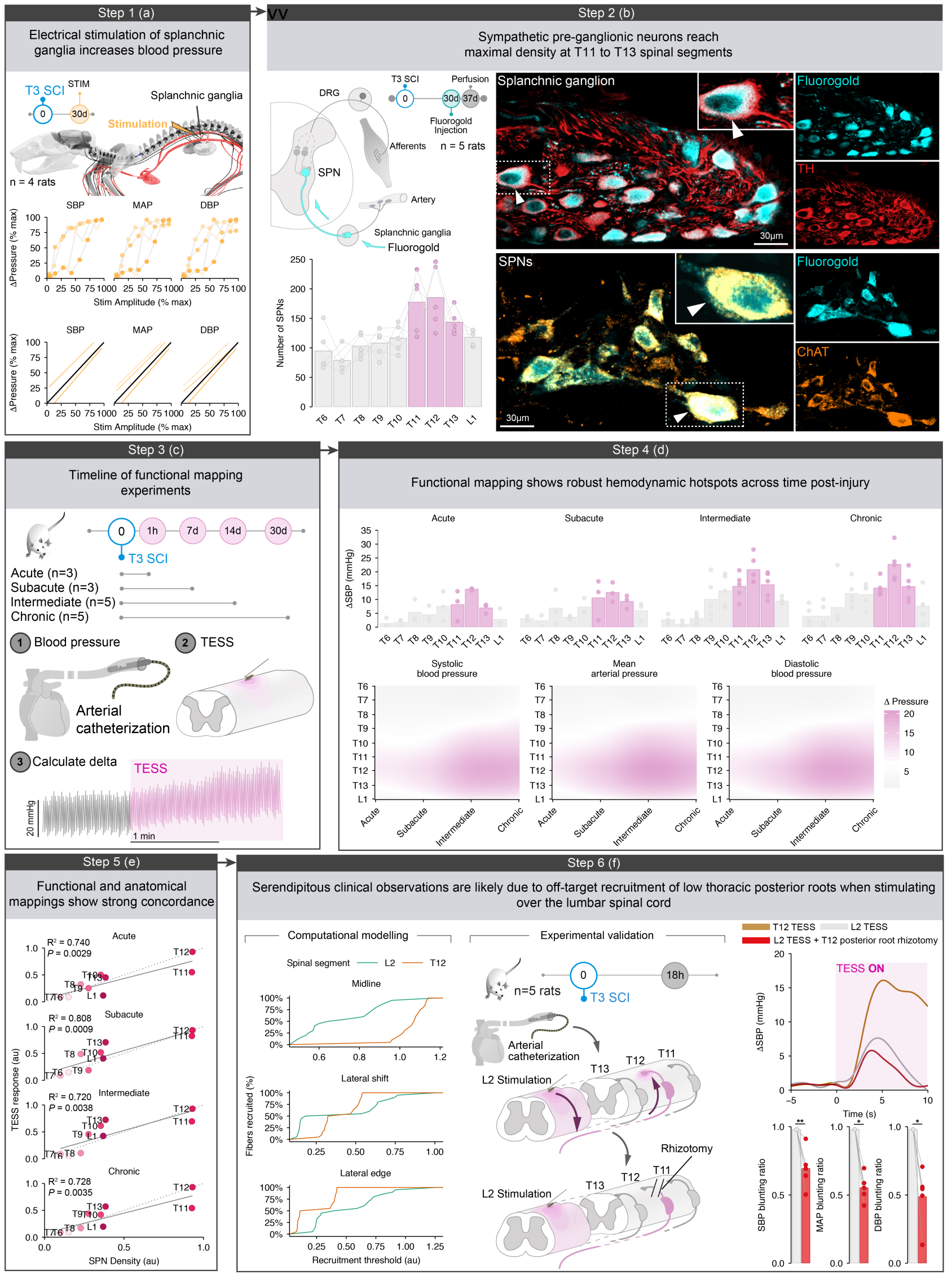




\section{Extended Data Figure 3. Functional and anatomical mapping reveal hemodynamic hotspots preferentially enriched to respond to epidural electrical stimulation.}

Step 1(a): We confirmed the role of the splanchnic ganglia in activating pressor responses. Electrical stimulation of splanchnic ganglia led to pressure responses that linearly increased with the stimulation amplitude $\left(n=4\right.$, linear mixed modelling; all $R^{2}>0.71$; all $\left.P<6.72 \mathrm{e}^{-12}\right)$. These relationships were robust across systolic blood pressure, diastolic blood pressure, and mean arterial pressure.

Step 2(b): Retrograde tracing of splanchnic ganglia using fluorogold labelled sympathetic pre-ganglionic neurons, which we confirmed using Choline Acetyltransferase (ChAT) staining. Successful injection was confirmed in splanchnic ganglia by colocalization of fluorogold with tyrosine hydroxylase (TH). We found a peak density in the number of sympathetic pre-ganglionic neurons projecting to splanchnic ganglia in the lower thoracic segments.

Step 3(c): We next completed functional mapping of the spinal cord by iterating through each segment and stimulating epidurally to activate pressor responses. We completed these experiments in animals one-hour post-injury (acute, $n=3$ ), one-week post-injury (sub-acute, $n=3$ ), two-weeks post-injury (intermediate, $n=$ 5), and four-weeks post-injury (chronic, $n=5$ ). We recorded blood pressure using a terminal carotid catheterization preparation, and calculated the peak change in blood pressure during the stimulation.

Step 4(d): We found that the response to targeted epidural electrical stimulation (TESS) increased with time post-injury, and that there was a hemodynamic hotspot in the lower thoracic spinal cord.

Step 5(e): We found a linear relationship between the functional and anatomical mapping results, providing a clear rationale for the existence of the observed hemodynamic hotspot (linear modelling; all $R^{2}>0.72$; all $P<0.003)$.

Step 6(f): We used computational modelling (see Extended Data Figure 4) and found that lateral edge (near root entrance/exit) placement of electrodes resulted in preferential recruitment of T12, even with TESS placed at L2. This was in contrast to more midline stimulation where we saw preferential recruitment off L2. Indeed, with electrodes placed with a subtle 'lateral shift' the shift in recruitment to T12 already begins. We reasoned this may be the mechanism by which serendipitous clinical observations were made. We confirmed this hypothesis using a rhizotomy experiment, whereby we cut the T12 root after stimulating with TESS at L2 $(n=5)$. We found a significant blunting of the TESS response in systolic blood pressure (paired one-tailed $\mathrm{t}$-test; $\mathrm{t}=-4.54 ; P=0.009$ ), mean arterial pressure (paired one-tailed $\mathrm{t}$-test; $\mathrm{t}=-3.08 ; P=0.027$ ), and diastolic blood pressure (paired one-tailed t-test; $\mathrm{t}=-2.40 ; P=0.048$ ). Bar plots represent the mean with raw data overlaid.

Abbreviations: ChAT: choline acetyltransferase; DBP: diastolic blood pressure; MAP: mean arterial pressure; SBP: systolic blood pressure; SCl: spinal cord injury; SPN: sympathetic pre-ganglionic neuron; TESS: targeted epidural electrical spinal cord stimulation; TH: tyrosine hydroxylase. 


\section{EXTENDED DATA FIGURE 4}

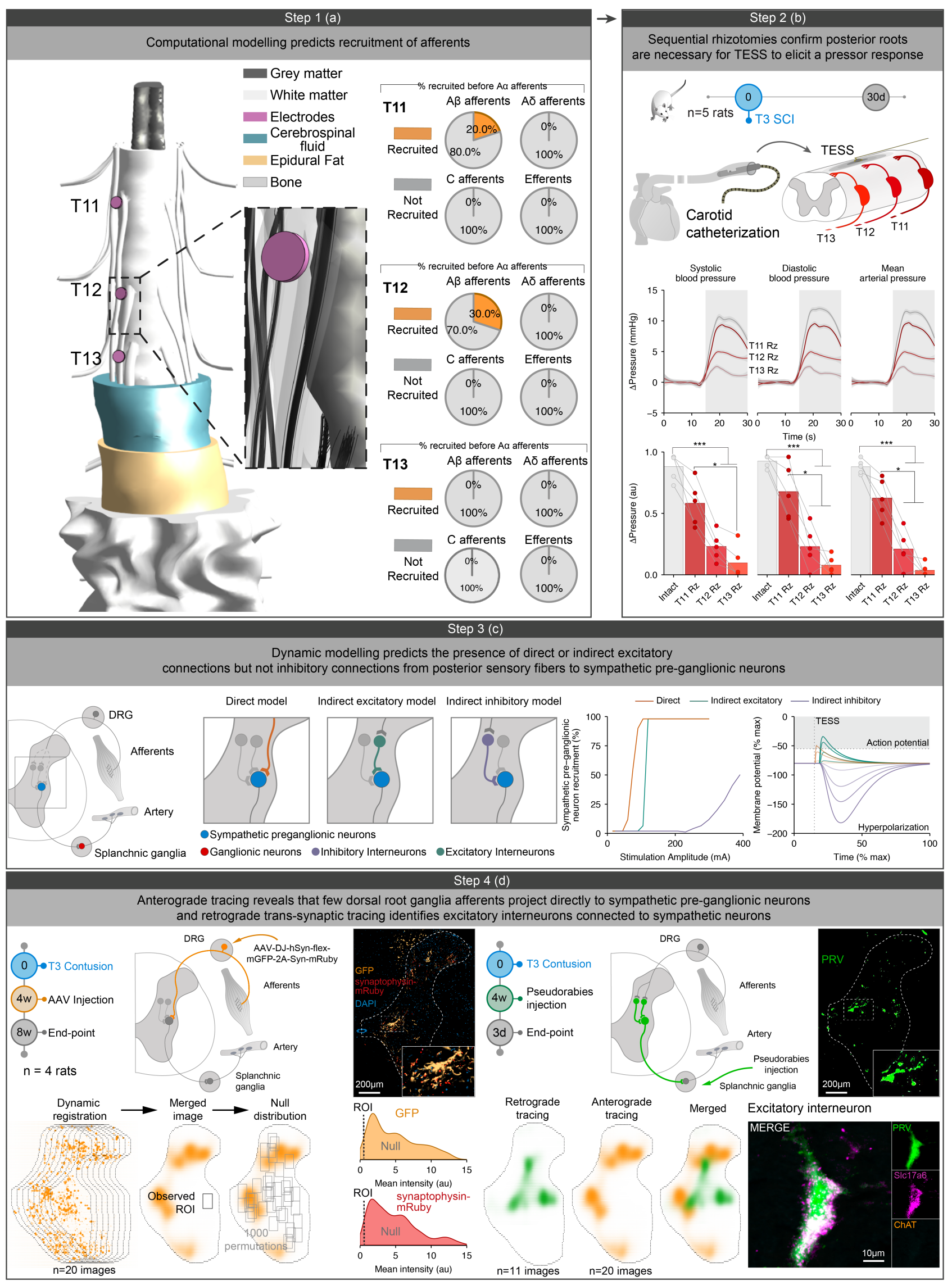




\section{Extended Data Figure 4. Epidural electrical stimulation induces pressor responses through the recruitment of posterior afferents and excitatory interneurons.}

Step 1(a): We developed a hybrid computational model based on real anatomical structures generated through high-resolution CT and MRI scans. This model combines a geometrically realistic 3D finite element model of the spinal cord with realistic compartmental cable models of all afferents, efferents and some interneurons. We established a computational pipeline to obtain anisotropic tissue property maps, discretize the model, perform simulations using an electro-quasi-static solver and couple these simulations with NEURON-based electrophysiology models (Sim4Life by ZMT, www.zurichmedtech.com). We investigated the recruitment patterns of various afferent and efferent fibers within the spinal cord structure. We found that stimulation over the dorsal aspect of the spinal cord led to high levels of recruitment of major afferents, prior to any recruitment of efferent neurons directly from the stimulation. This suggested that epidural electrical stimulation activates pressor responses by recruiting afferents.

Step 2(b): Next, we experimentally tested the hypothesis that pressor responses induced by TESS were dependent on afferent activation. We completed successive dorsal rhizotomies at T11, T12, and T13 and found a graded reduction in the response to stimulation (one-way ANOVA; all $P<0.001$; post-hoc results indicated), with the largest decrease when removing T12, consistent with our functional and anatomical mapping results. Grey box indicates stimulation. Bar plots represent the mean with raw data overlaid.

Step 3(c): Next, we developed a NEURON-based spiking neural network model composed of integrate-andfire neurons to predict the presence of direct, indirect excitatory, and indirect inhibitory connections. Indirect inhibitory connections resulted in poor sympathetic pre-ganglionic neuron recruitment (left panel) and in the minimization of membrane potentials in response to increasing stimulation amplitude (right panel; various stimulation amplitudes indicated by alpha; action potential threshold indicated by horizontal dotted line; stimulation onset indicated by vertical dotted line). This suggested that pressor responses to TESS likely are mediated by either direct, monosynaptic connections between afferents and sympathetic pre-ganglionic neurons or by indirect circuits including excitatory interneurons.

Step 4(d): We completed anterograde tracing of the dorsal root ganglia. Using dynamic image registration we generated a digital dorsal horn whereby we could select a region of interest (ROI; grey box) and determine the mean intensity ('Observed $\mathrm{ROI}^{\prime}$ ) of either axons (orange) or synapses (red). Using 1000 bootstraps of random ROls as a null distribution we found a depletion of axons (empirical $P=0.019$ ) and synapses (empirical $P=0.001$ ) in the intermediolateral column. We confirmed this result by counting neurons with appositional synapses on ChATON neurons in the ventral horn versus the lateral horn and found a similar statistical depletion ( $n=10$ images, 294 neurons; Fisher's exact test; OR: $0.082 ; P<2.2 \mathrm{e}^{-16}$ ). This suggested that the most likely circuit mediating these responses instead included an excitatory interneuron. We therefore completed retrograde trans-synaptic tracing and found interneurons trans-synaptically connected to splanchnic ganglia that were Slc17a6 positive, and had Vglut1 synaptic puncta in their immediate vicinity (see Figure 2), suggesting direct connections with large diameter afferents.

${ }^{\star} P<0.05 ;{ }^{* \star} P<0.01 ;{ }^{* \star \star} P<0.001$.

Abbreviations: ChAT: choline acetyltransferase; PRV: pseudorabies; ROI: region of interest; Rz: rhizotomy; TESS: targeted epidural electrical spinal cord stimulation. 


\section{EXTENDED DATA FIGURE 5}
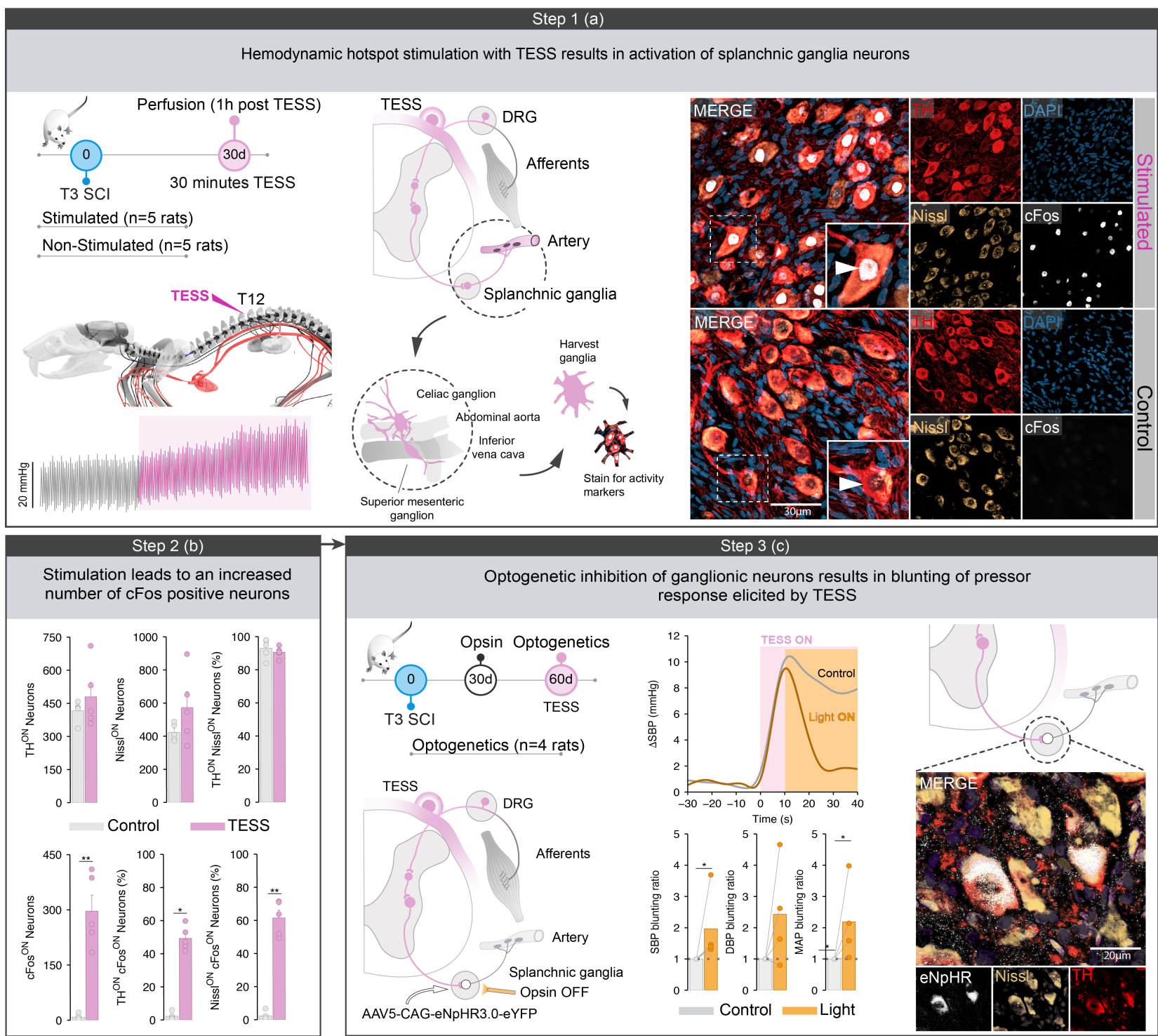

Step 3 (c)
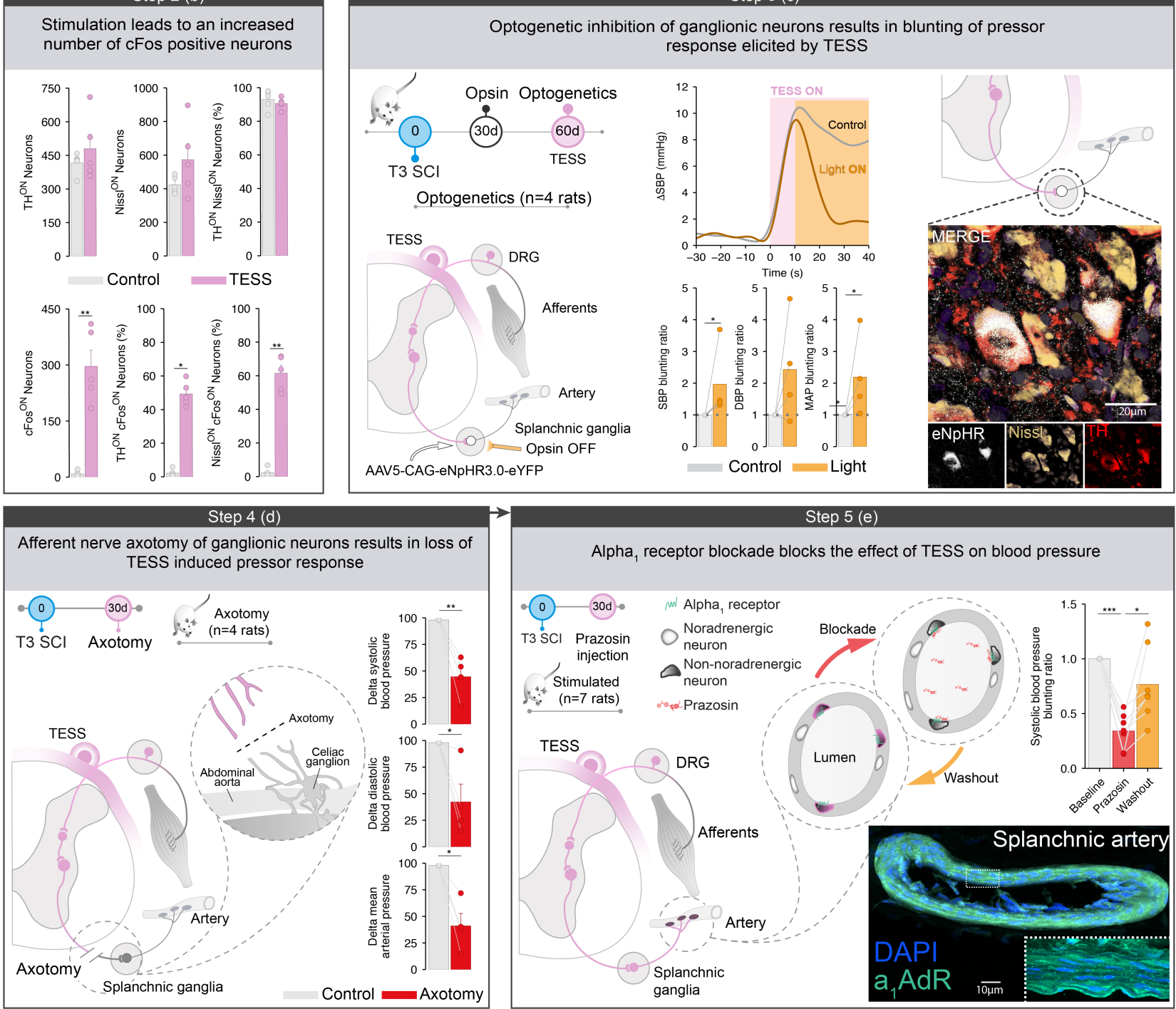


\section{Extended Data Figure 5. Epidural electrical stimulation induces pressor responses through the recruitment of splanchnic ganglia.}

Step 1(a): To determine whether epidural electrical stimulation activated splanchnic ganglia we stimulated rats with T3 spinal cord injury (SCl) for thirty minutes $(n=5)$, or did not stimulate them $(n=5)$. We harvested the splanchnic ganglia and subjected them to immunohistochemisty. We stained each section for tyrosine hydroxylase (TH), cFos (immediate early gene), DAPI, and Nissl to confirm neuronal phenotypes. We then used an automatic cell segmentation algorithm to identify cells that coexpressed TH, Nissl, and or cFos.

Step 2(b): Quantifications revealed that animals stimulated with thirty minutes of EES had more cFos positive neurons than unstimulated rats (independent samples one-tailed t-test; $\mathrm{t}=6.56 ; P=0.001$ ), and that these neurons colocalized with TH and Nissl stains (both $p<0.001$ ).

Step 3(c): We next completed two loss of function studies to examine the causal role of the splanchnic ganglia in the pressor responses to TESS. We conducted an optogenetics silencing experiment $(n=4)$ and an axotomy experiment whereby we surgically severed the connection between the sympathetic preganglionic neurons in the spinal cord and the splanchnic ganglia $(n=4)$. We completed these experiments on animals 60 days after T3 spinal cord injury. For optogenetics experiments viral injections occurred at 30 days post-injury. Activating the inhibitory opsin eNpHr3.0 with yellow light suppressed the pressor response to TESS, and therefore increased the difference between the peak response to TESS and the minimum response during light (or control) for systolic blood pressure (independent samples one-tailed $\mathrm{t}$-test; $\mathrm{t}=$ 2.90; $P=0.031$ ) and mean arterial pressure (independent samples one-tailed $t$-test; $t=2.60 ; P=0.040$ ).

Step 4(d): Axotomy of the connection between sympathetic pre-ganglionic neurons and splanchnic ganglia likewise blunted the pressor response for systolic blood pressure (independent samples one-tailed t-test; $\mathrm{t}$ $=-4.54 ; P=0.001$ ), diastolic blood pressure (independent samples one-tailed t-test; $\mathrm{t}=-2.40 ; P=0.048$ ), and mean arterial pressure (independent samples one-tailed t-test; $\mathrm{t}=-3.08 ; P=0.027$ ). These experiments indicate the splanchnic ganglia are necessary to induce a pressor response with TESS.

Step 5(e): We next tested whether blocking alpha 1 receptors on systemic blood vessels would blunt the response to TESS. We administered IV prazosin and found an immediate loss of pressor responses to TESS. The response returned after drug washout (one-way repeated measures ANOVA; $F_{2}=15.63 ; P=0.0001$; Tukey HSD).

${ }^{\star} P<0.05 ;{ }^{* \star} P<0.01 ; * \star * P<0.001$.

Abbreviations; DRG: dorsal root ganglia; SBP: systolic blood pressure; TESS: targeted epidural electrical spinal cord stimulation; TH: tyrosine hydroxylase. 


\section{EXTENDED DATA FIGURE 6}

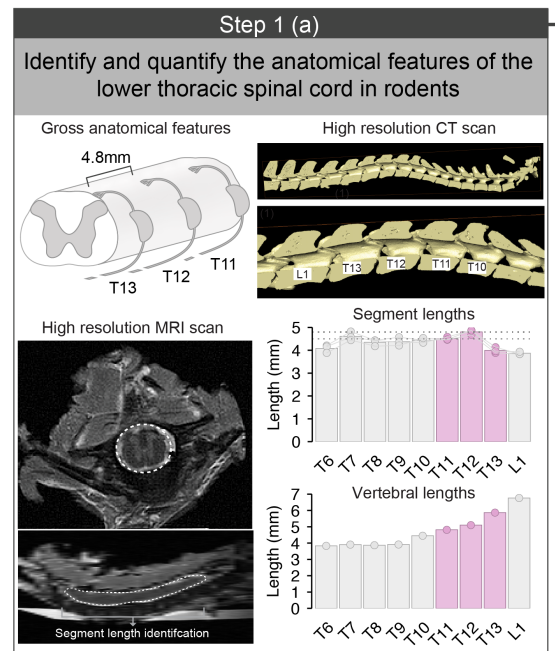

Step 4 (d)

Natural system dynamics were revealed through concomitant recordings of spiking activity in the rostral ventrolateral medulla (RVLM), sympathetic nerve activity in the spinal cord, and arterial blood pressure

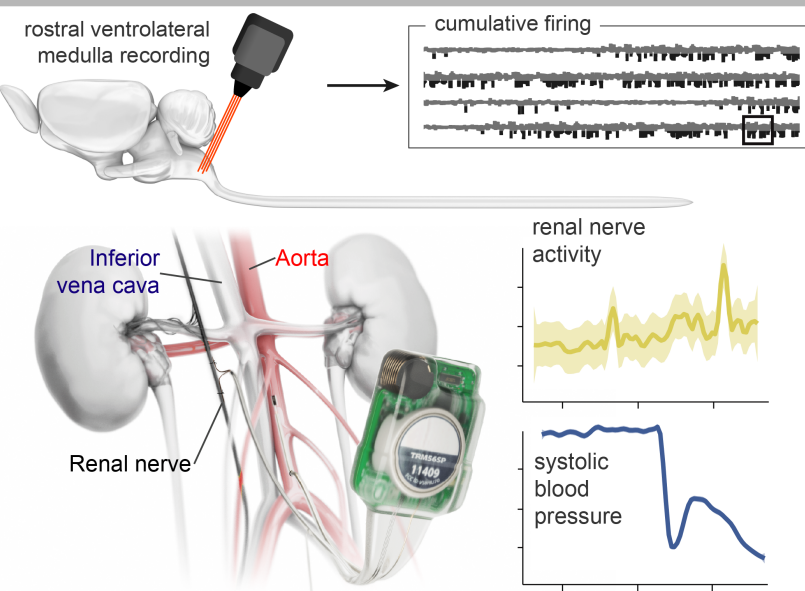

Step 6 ( $f$ )

Coordinated dynamics between blood pressure, the RVLM, and sympathetic nerve activity are quantified using regression

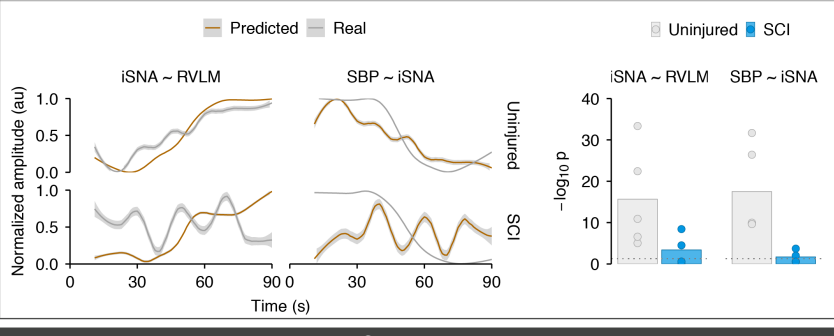

Step $7(\mathrm{~g})$

The RVLM delivers an efferent volley with a clear delay between hemodynamic hotspots

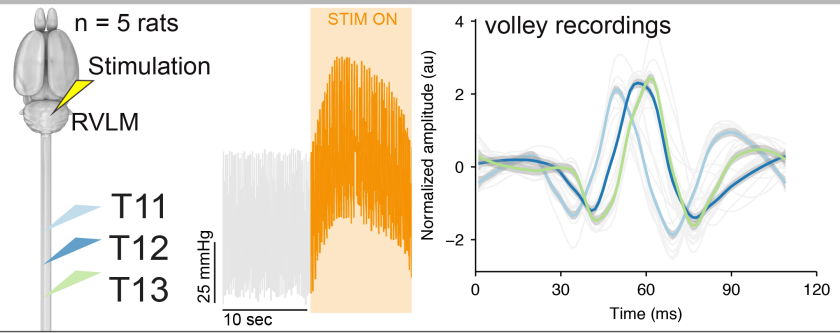

Step 2 (b)

Design and fabricate an electrode array specifically targeting hemodynamic hotspots
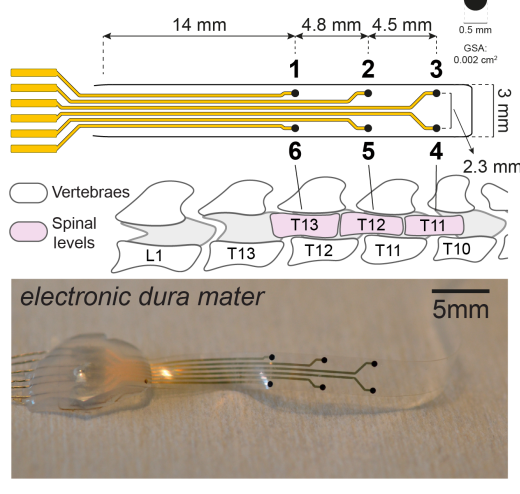

Electrochemical signature of targeted e-dura and blood pressure increase following TESS
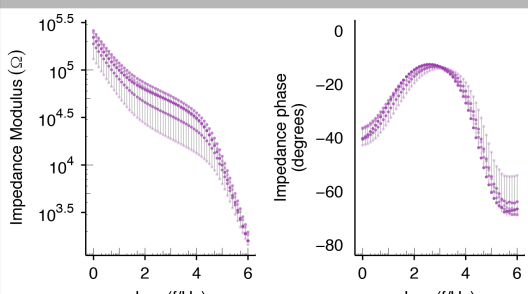

$\log (f / \mathrm{Hz})$

TESS ON

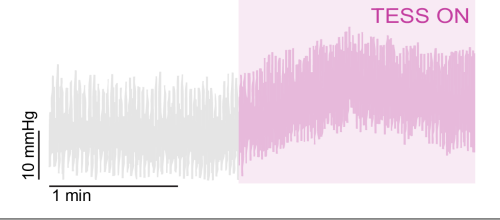

Step 5 (e)

Coordinated dynamics between blood pressure, the RVLM, and sympathetic nerve activity are disrupted after spinal cord injury
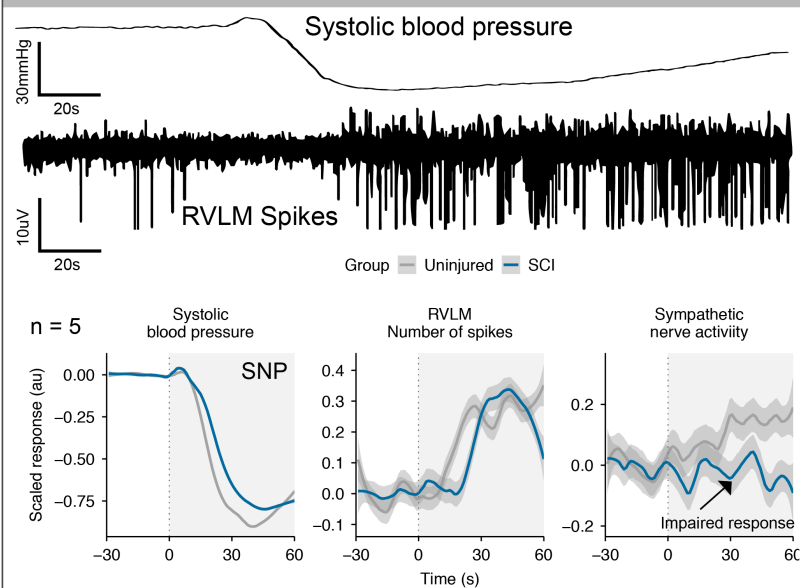

Step $8(\mathrm{~h}$
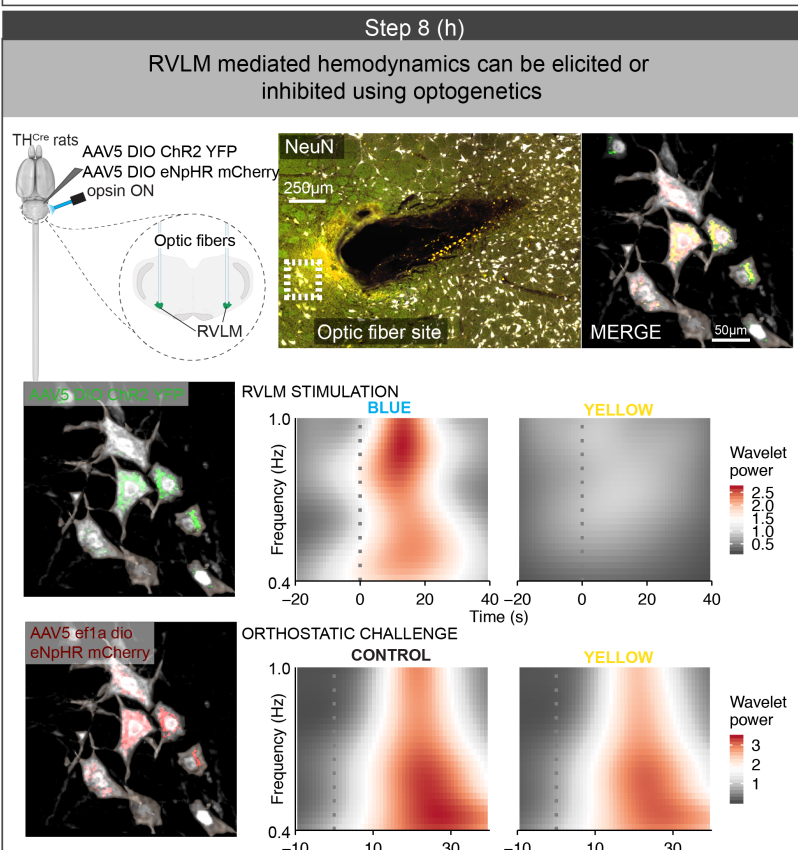

Wavelet

2.5 1.0
3
0.5

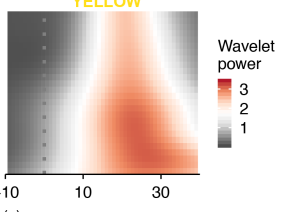




\section{Extended Figure 6. Mechanistic insight enabled the development of a targeted electronic dura mater and biomimetic stimulation strategy to recapitulate the natural dynamics of the sympathetic nervous system.}

Step 1(a): To develop the spatial features of an electrode array specifically targeting hemodynamic hotspots we first quantified all the features of the low thoracic spinal column. We measured the precise length of each spinal segment and vertebrae using a combination of gross anatomical dissections, high resolution CT scans, and custom MRI sequences.

Step 2(b): We used these anatomical features, driven by the identification of hemodynamic hotspots to develop an electronic dura mater specifically targeting T11, T12, and T13 spinal segments. Detailed dimensions of the active stimulation sites $\left(500 \mu \mathrm{m}\right.$ diameter, $0.002 \mathrm{~cm}^{2}$ geometric surface area).

Step 3(c): When placed on the spinal cord, the array increases blood pressure and all electrodes are functional. Top: electrochemical impedance spectrum (modulus, left and phase, right) of an electrode array acquired in vitro post-fabrication.

Step 4(d): To recapitulate the natural dynamics of the sympathetic nervous system we first measured from three key areas including the rostral ventrolateral medulla (RVLM) using a multichannel neural signal processor, the renal sympathetic nerve, and blood pressure using an arterial cannula.

Step 5(e): We measured these three key areas in response to a hypotensive stimulus (sodium nitroprusside [SNP]) in both injured and uninjured animals $(n=5)$. We found that there was an impaired response in the sympathetic nerve activity after $\mathrm{SCl}$.

Step 6(f): To quantify the changes in these dynamics we trained a feed-forward neural network to predict a continuous output from a given input. For example, predicting sympathetic nerve activity (iSNA) from RVLM activity (i.e., iSNA RVLM). We found that in uninjured animals there were strong correlations between each step in the system, whereby the model could predict one from the other. Here, we show the ability of the model to predict iSNA from RVLM spikes, and to predict SBP from iSNA (strength of correlation [Pearson correlation] presented as $-\log 10(p)$ for each group and comparison). Of note is that changes in the system are driven by an external perturbation and therefore our model quantifies simply the relative dynamics of this response.

Step 7(g): To understand the timing delay of RVLM activation to sympathetic outflow from the spinal cord we stimulated the RVLM electrically, and measured the efferent volley over T11, T12, and T13 $(n=5)$. Stimulation of the RVLM dramatically increased blood pressure, confirming localization of the stimulation. We then measured the delay between action potentials in response to $100 \mathrm{~Hz} 10$ s pulse trains of RVLM stimulation and found a $2.5 \pm 0.4 \mathrm{~ms}$ delay between segments. Representative traces across segments are shown for one animal. We therefore integrated this delay into the stimulation design between segments. Step 8 (h): Finally, to understand the precise role of frequency dynamics in blood pressure control we stimulated the RVLM using optogenetics in TH-Cre rats. We found that stimulation with blue light led to a robust increase in wavelet spectrogram within the $0.4-1.0 \mathrm{~Hz}$ band (paired samples one-tailed $\mathrm{t}$-test; $\mathrm{t}=2.67$; $P=0.028$ ). This was in contrast to activation of an inhibitory opsin using a yellow laser (which would, in this case, inhibit the RVLM due to the presence of an inhibitory opsin), which showed significantly less activation compared to blue light (paired samples one-tailed $t$-test; $t=2.44 ; P=0.035$ ). In response to an orthostatic challenge, the wavelet power response in uninjured rats was less pronounced in the presence of inhibitory (yellow) light (bottom panels; independent samples one-tailed t-test; $t=4.04 ; P=0.008$ ).

${ }^{\star} P<0.05 ;{ }^{\star \star} P<0.01 ;{ }^{\star \star \star} P<0.001$.

Abbreviations: iSNA: integrated sympathetic nerve activity; RVLM: rostral ventrolateral medulla; SBP: systolic blood pressure; TESS: targeted epidural electrical spinal cord stimulation; TH: tyrosine hydroxylase. 


\section{EXTENDED DATA FIGURE 7}

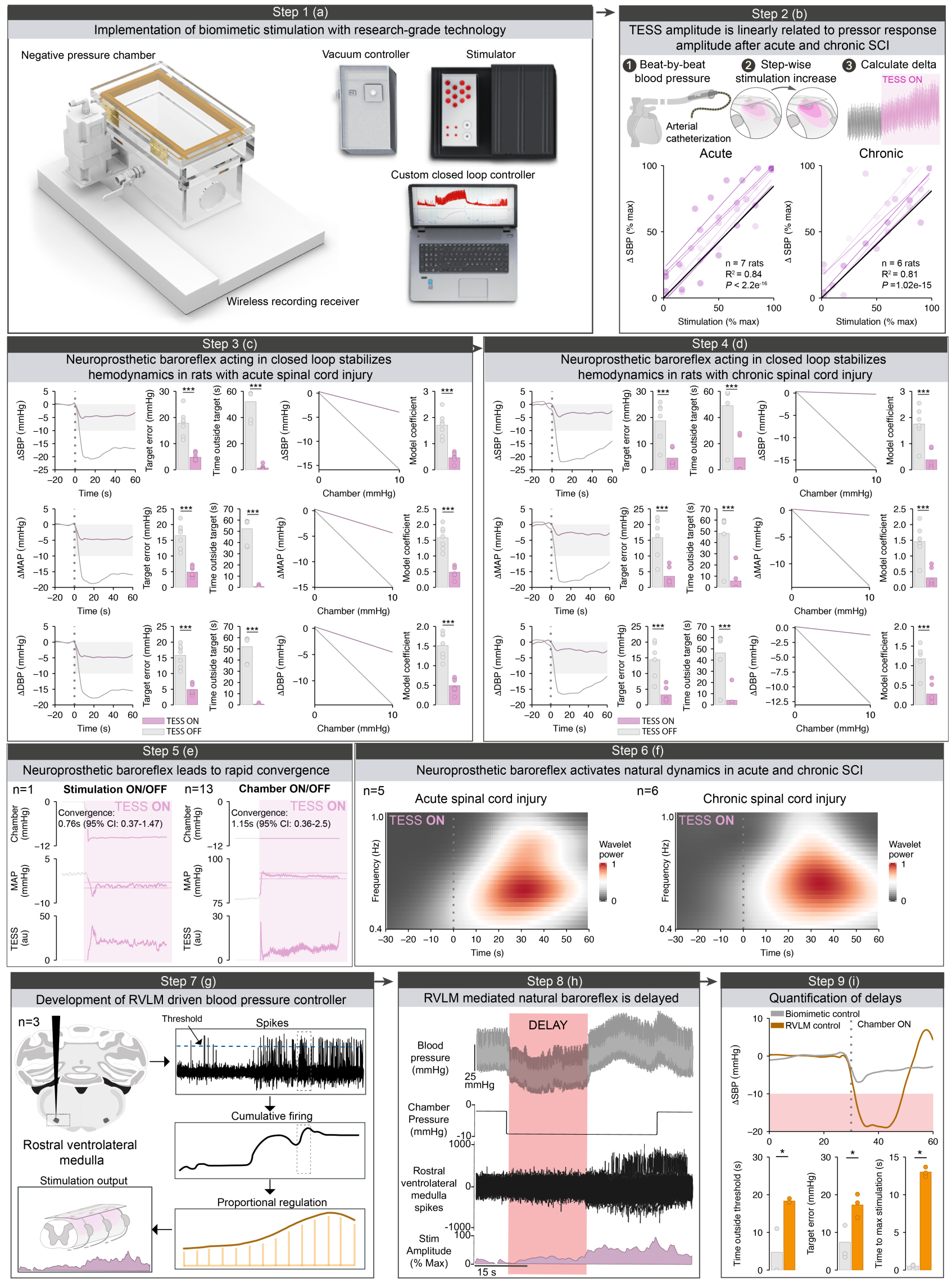




\section{Extended Data Figure 7. Neuroprosthetic baroreflex implemented in rodents with SCI.}

Step 1(a): We tested whether we could stabilize hemodynamics using the neuroprosthetic baroreflex, operating in closed-loop, in animals with acute $(n=7 ; 18$ hours post-injury) and chronic $(n=6$; one month post-injury) T3 spinal cord injury (SCl). We implemented the neuroprosthetic baroreflex within researchgrade technology in order to achieve precise control over stimulation parameters.

Step 2(b): We found a linear relationship between stimulation amplitude and the pressor response to stimulation in both animals with acute (mixed model linear regression; $R 2=0.84, P<2.2 e^{-16}$ ) and chronic (mixed model linear regression; R2 $=0.81, P<1.0 \mathrm{e}^{-15}$ ) spinal cord injury.

Step 3(c): We completed a series of trials to test the ability of the neuroprosthetic baroreflex to stabilize hemodynamics in rats with acute spinal cord injury. The neuroprosthetic baroreflex was activated in closedloop prior to the activation of the lower-body negative pressure chamber. In trials where the stimulation was $\mathrm{ON}$, we found a reduction in the target (baseline) error (paired samples one-tailed t-tests; SBP: $\mathrm{t}=-6.12, P$ $\left.=5.50 \mathrm{e}^{-04} ; \mathrm{MAP} ; \mathrm{t}=-6.08, P=4.48 \mathrm{e}^{-04} ; \mathrm{DBP} ; \mathrm{t}=-5.85, P=4.34 \mathrm{e}^{-04}\right)$ reduced time outside key thresholds $(-$ $10 \mathrm{mmHg}$; paired samples one-tailed t-tests; SBP: $\mathrm{t}=-12.52, P=9.94 \mathrm{e}^{-06} ; \mathrm{MAP} ; \mathrm{t}=-12.29, P=8.83 \mathrm{e}^{-06} ; \mathrm{DBP}$; $\left.t=-11.73, P=1.15 e^{-05}\right)$, a restoration of the non-linear relationship between blood pressure and chamber pressure, and a concomitant reduction in the linear model coefficient (likelihood ratio test of nested models; all $P<0.001$ ). These quantifications held for systolic blood pressure (top), diastolic blood pressure (middle), and mean arterial pressure (bottom).

Step 4(d): We completed the exact same experiments on animals with chronic spinal cord injury and found similar results to those of the acutely injured rats. Specifically, in trials where the stimulation was ON, we found a reduction in the target (baseline) error (paired samples one-tailed t-tests; SBP: $\mathrm{t}=-3.84, P=0.006$; MAP; $\mathrm{t}=-3.83, P=0.006 ; \mathrm{DBP} ; \mathrm{t}=-3.83, P=0.006)$, reduced time outside key thresholds $(-10 \mathrm{mmHg}$; paired samples one-tailed t-tests; SBP: $\mathrm{t}=-4.37, P=0.004 ; \mathrm{MAP} ; \mathrm{t}=-4.43, P=0.003 ; \mathrm{DBP} ; \mathrm{t}=-4.21, P=0.004)$, a restoration of the non-linear relationship between blood pressure and chamber pressure, and a concomitant reduction in the linear model coefficient (likelihood ratio test of nested models; all $P<0.001$ ). These quantifications held for systolic blood pressure (top), diastolic blood pressure (middle), and mean arterial pressure (bottom).

Step 5(e): We found that in response to stimulation blood pressure rapidly reached the set-point, with convergence times of $0.76 \mathrm{~s}$ in the example case presented in Figure 4, and $1.15 \mathrm{~s}$ (95\% Cl: $0.36-2.5 \mathrm{~s}$ ) across $n=13$ animals in response to the negative pressure chamber. In this case convergence was defined as stable within $2.5 \mathrm{mmHg}$.

Step 6(f): The neuroprosthetic baroreflex, acting in closed loop, re-established natural frequency dynamics (increased wavelet power in the $0.4-1.0 \mathrm{~Hz}$ spectrogram) in both animals with acute (paired samples onetailed t-test; $\mathrm{t}=4.46 ; P=0.002$ ) and chronic $\mathrm{SCl}$ (paired samples one-tailed $\mathrm{t}$-test; $\mathrm{t}=3.37 ; P=0.014$ ).

Step 7(g): We tested how our neuroprosthetic baroreflex compared to the natural baroreflex by recording from the rostral ventrolateral medulla and using this as input to our controller $(n=3)$, instead of automated blood pressure changes as the detection (i.e., a brain-spine interface). We scaled the stimulation amplitude in proportion to the number of detected spikes in the rostral ventrolateral medulla.

Step 8(h): We found a 10-20s delay in the response within the rostral ventrolateral medulla in response to a negative pressure stimulus. In contrast, the neuroprosthetic baroreflex responded more than 10x faster to stabilize hemodynamics in the face of an orthostatic challenge.

Step 9(i): Quantifications of Step 8 (h); Wilcoxon rank sum test all $P<0.05$ ).

${ }^{\star} P<0.05 ;{ }^{* \star} P<0.01 ; * \star \star x<0.001$.

Abbreviations: DBP: diastolic blood pressure; MAP: mean arterial pressure; RVLM: rostral ventrolateral medulla; SBP: systolic blood pressure; SBP: systolic blood pressure; SCI: spinal cord injury; TESS: targeted epidural electrical spinal cord stimulation. 


\section{EXTENDED DATA FIGURE 8}

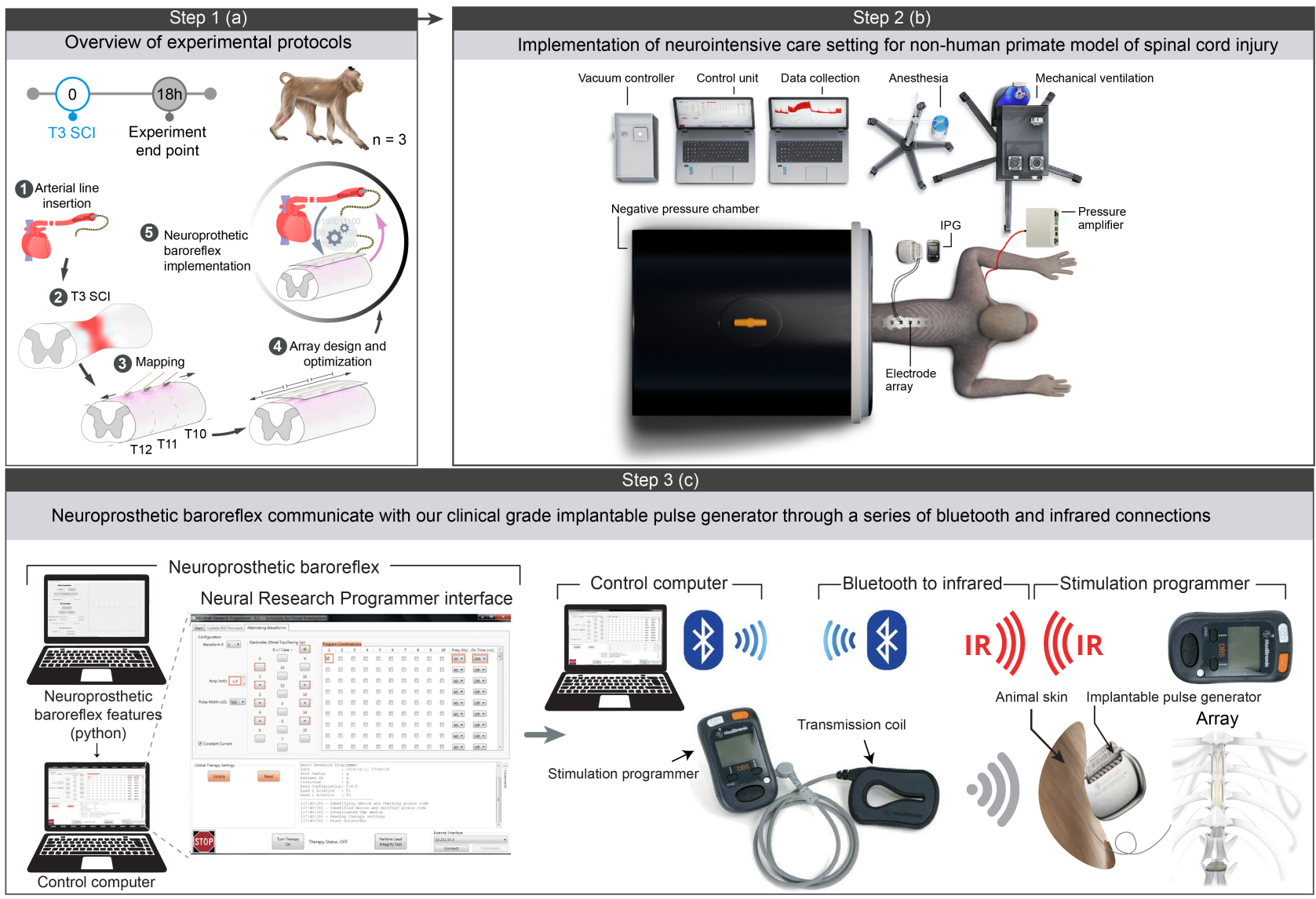

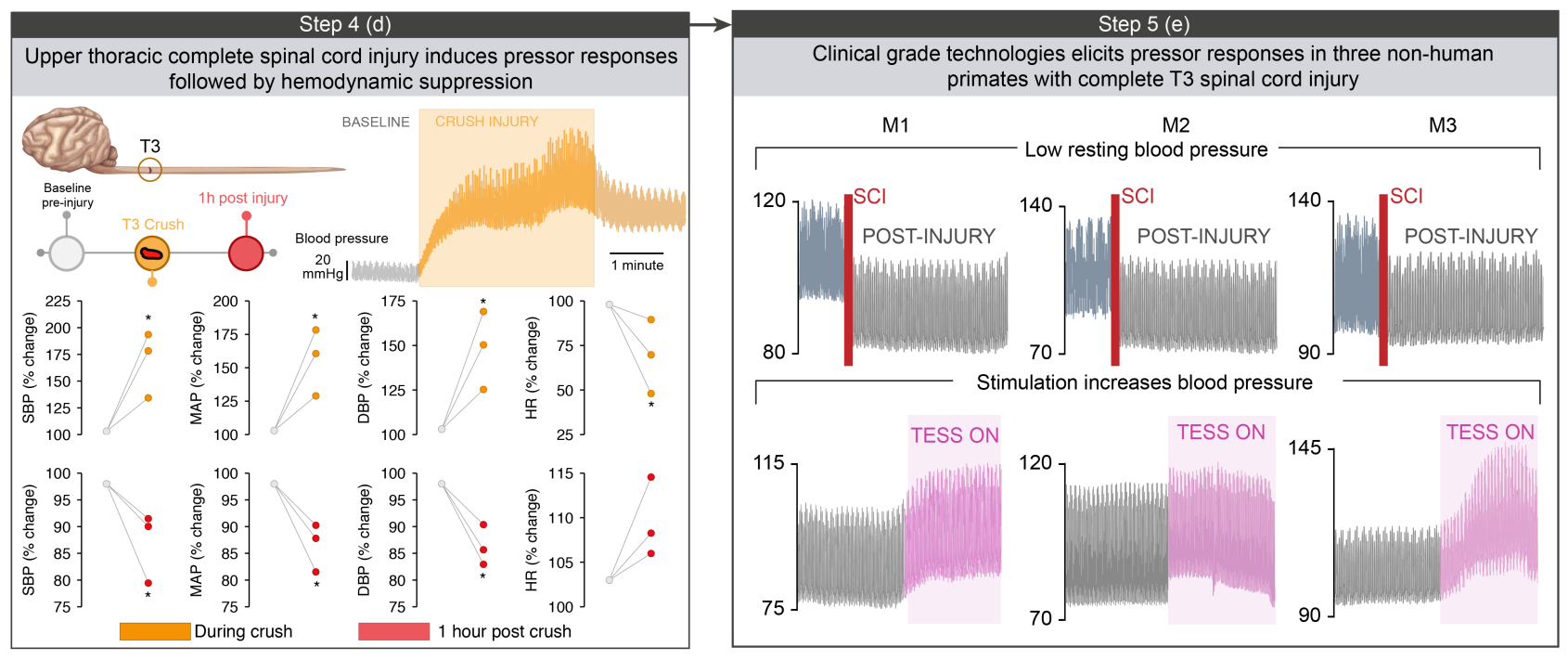




\section{Extended Data Figure 8. Translation of the neuroprosthetic baroreflex to non-human primates.}

Step 1(a): To further establish the efficacy of the neuroprosthetic baroreflex we performed experiments in three non-human primates. First, we measured arterial blood pressure using an invasive catheter in the subclavian artery. Next, we performed a T3 spinal cord injury (SCl) in order to mimic the experimental conditions of our rodent experiments. We next mapped the pressor responses to epidural stimulation from T7 to L3. We combined these results with ex-vivo dissections of the anatomical dimensions of the lower thoracic spinal cord in Rhesus Macaques $(n=3)$ to design an electronic dura mater. Finally, we implemented all the features of the neuroprosthetic baroreflex.

Step 2(b): We tested the efficacy of the neuroprosthetic baroreflex specifically within the context of acute traumatic $\mathrm{SCl}$. We emulated all the features of standard neurointensive care including arterial blood pressure measurements, clinical grade anesthesia (intravenous Propofol), as well as temperature and respiration control. We integrated our stimulation approach into clinical-grade technologies using an implantable pulse generator and a spatially selective spinal implant.

Step 3(c): All the features of the neuroprosthetic baroreflex were injected into our previously used clinicalgrade stimulation approach. Briefly, the neuroprosthetic baroreflex received beat-by-beat continuous blood pressure in order to provide closed loop control. Stimulation output control was sent to the neural research programmer interface, which communicates with the implantable pulse generator through a series of bluetooth and infrared links. These commands were then sent directly to the customized spinal implant.

Step 4(d): Similar to rodent experiments, we found that T3 spinal cord injury induced a significant surge in systolic blood pressure (paired one-tailed t-test; $t=4.15 ; P=0.027$ ), mean arterial pressure (paired onetailed t-test; $\mathrm{t}=3.96 ; P=0.029$ ), diastolic blood pressure (paired one-tailed t-test; $\mathrm{t}=3.80 ; P=0.031$ ), and an accompanying decrease in heart rate (paired one-tailed t-test; $t=-4.05 ; P=0.028$ ). By one-hour postinjury we observed clinically relevant neurogenic shock, characterized by decreased systolic blood pressure (paired one-tailed t-test; $\mathrm{t}=-3.20 ; P=0.043$ ), mean arterial pressure (paired one-tailed $\mathrm{t}$-test; $\mathrm{t}=-5.23 ; P=$ 0.017 ), and diastolic blood pressure (paired one-tailed t-test; $\mathrm{t}=-6.24 ; P=0.012$ ).

Step 5(e): Despite the fact that we observed an immediate decrease in resting blood pressure, epidural electrical stimulation was able to cause an immediate and transient pressor response in all three animals. In all cases, where percent change is presented statistics were completed on raw values.

${ }^{\star} P<0.05 ;{ }^{\star \star} P<0.01 ;{ }^{\star \star \star} P<0.001$.

Abbreviations: DBP: diastolic blood pressure; EES: epidural electrical stimulation HR: heart rate; MAP: mean arterial pressure; SBP: systolic blood pressure; SCl: spinal cord injury. 


\section{EXTENDED DATA FIGURE 9}

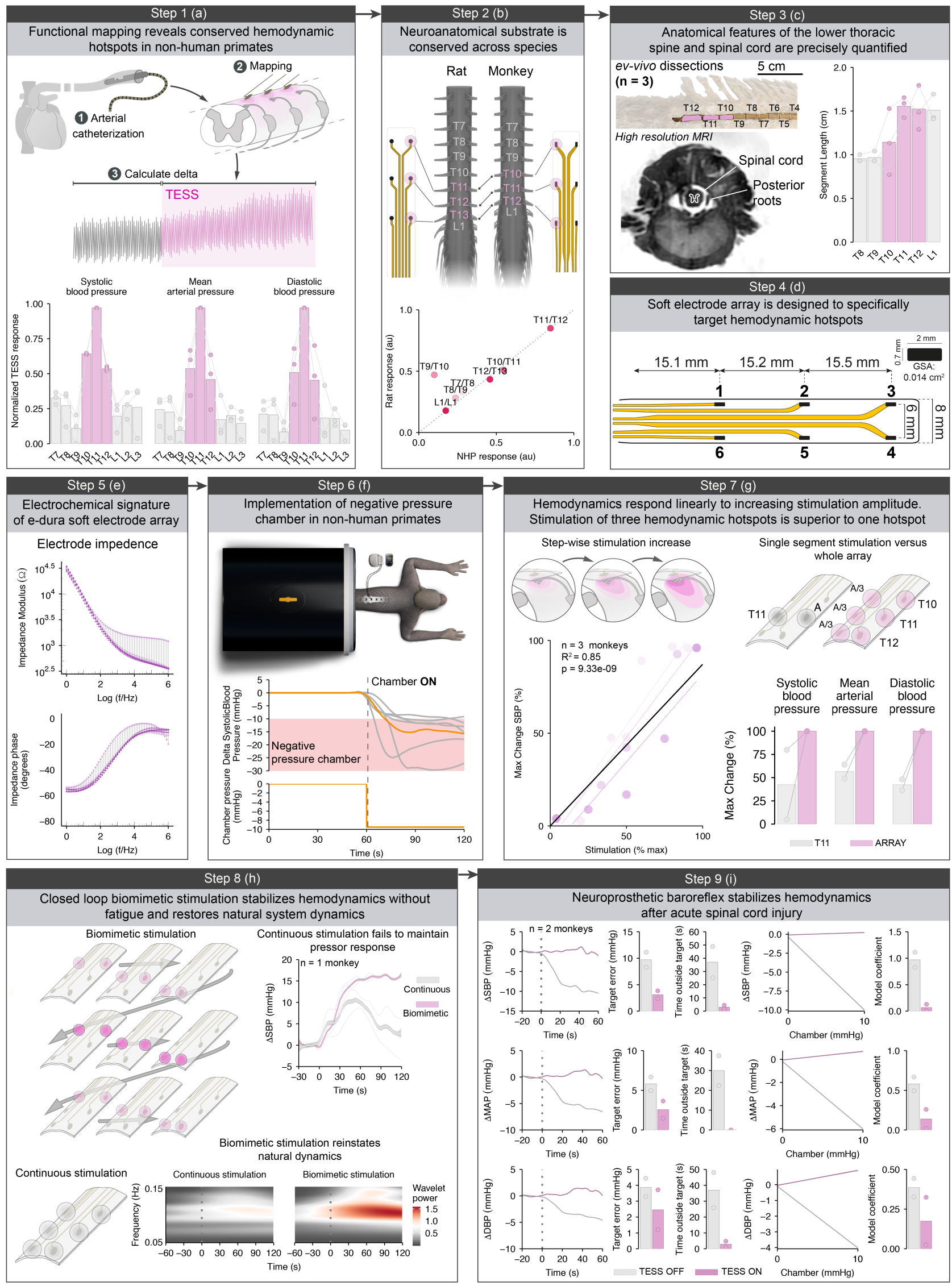




\section{Extended Data Figure 9. Neuroprosthetic baroreflex implemented in non-human primates with spinal cord injury.}

Step 1(a): We recorded blood pressure using an axillary artery catheterization preparation, and calculated the peak change in blood pressure during stimulation. We found that there was a hemodynamic hotspot in the lower thoracic spinal cord, specifically over segments T10, T11, and T12.

Step 2(b): Accounting for the offset in segments between species, we found a linear relationship between the functional mapping results in rats and the functional mapping results in non-human primates, further confirming the localization of a hemodynamic hotspot in the last three thoracic segments (linear modelling; $\mathrm{R}^{2}=0.65 ; P=0.018$.

Step 3(c): To develop the spatial features of an electrode array specifically targeting hemodynamic hotspots in the non-human primate we quantified all the features of the low thoracic spinal column. We measured the precise length of each spinal segment and vertebra using a combination of gross anatomical dissections, high resolution CT scans, and custom MRI sequences.

Step 4(d): We used these anatomical features, driven by the identification of hemodynamic hotspots to develop an electronic dura mater specifically targeting T10, T11, and T12 spinal segments. Detailed dimensions of the active stimulation sites $\left(0.7 \mathrm{~mm}\right.$ diameter, $0.014 \mathrm{~cm}^{2}$ geometric surface area).

Step 5(e): Electrochemical impedance spectrum (modulus, left and phase, right) of an electrode array acquired in vitro post-fabrication indicates functional, low-impedance electrodes.

Step 6(f): We fully implemented a negative pressure chamber designed to fit a non-human primate in order to induce an orthostatic challenge. We found that stimulation using the chamber led to an immediate and consistent decrease in blood pressure.

Step 7(g): We next implemented our biomimetic stimulation protocols. We found a linear relationship between stimulation amplitude and the pressor response $(\mathrm{n}=3$ monkeys; linear modelling, $\mathrm{R} 2=0.85 ; P=$ $\left.9.33 \mathrm{e}^{-05}\right)$. Compared to stimulating only one hotspot, stimulating with all three sets of electrodes, and therefore targeting each hemodynamic hotspot led to a more robust increase in blood pressure $(n=2$ monkeys).

Step 8(h): Implementation of the neuroprosthetic baroreflex, acting in closed loop, led to sustained increases in blood pressure that did not fatigue ( $n=1$ monkey). In comparison, continuous open-loop stimulation elicited an increase in blood pressure that was immediately followed by rapid fatigue. Only biomimetic stimulation re-established the natural frequency dynamics, revealed using wavelet decomposition ( $\mathrm{n}=2$ monkeys).

Step 9(i): Using this closed loop approach, we found a reduction in the target (baseline) error, reduced time outside key thresholds $(-5 \mathrm{mmHg} ;-2 \mathrm{mmHg}$ for diastolic blood pressure), a restoration of the non-linear relationship between blood pressure and chamber pressure, and a concomitant reduction in the linear model coefficient. These quantifications held for systolic blood pressure (top), diastolic blood pressure (middle), and mean arterial pressure (bottom).

Abbreviations: DBP: diastolic blood pressure; MAP: mean arterial pressure; SBP: systolic blood pressure; TESS: targeted epidural electrical spinal cord stimulation. 


\section{EXTENDED DATA FIGURE 10}

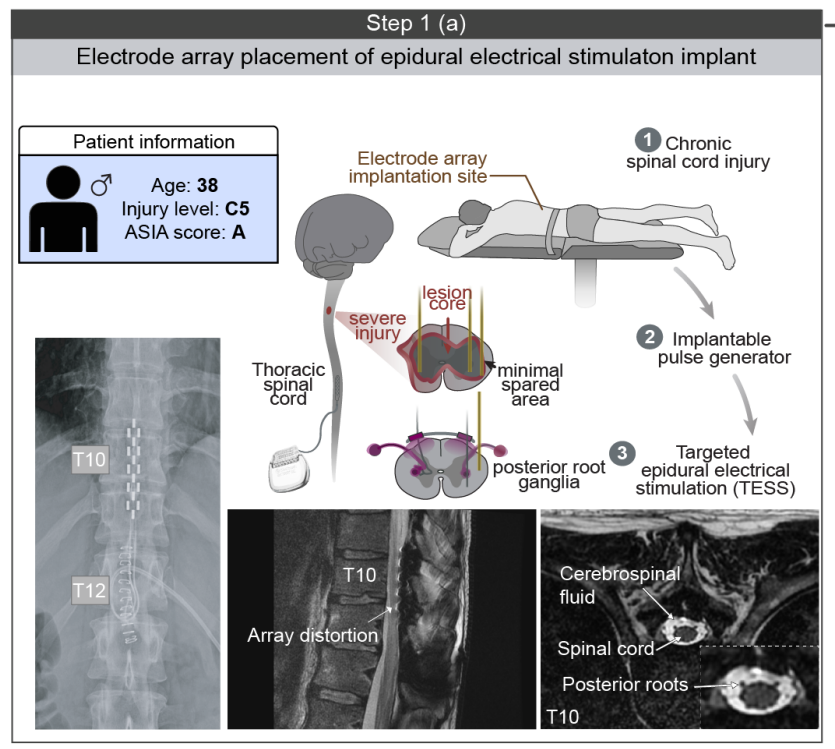

Step 2 (b)

Experimental protocols and spatial configuration assessments
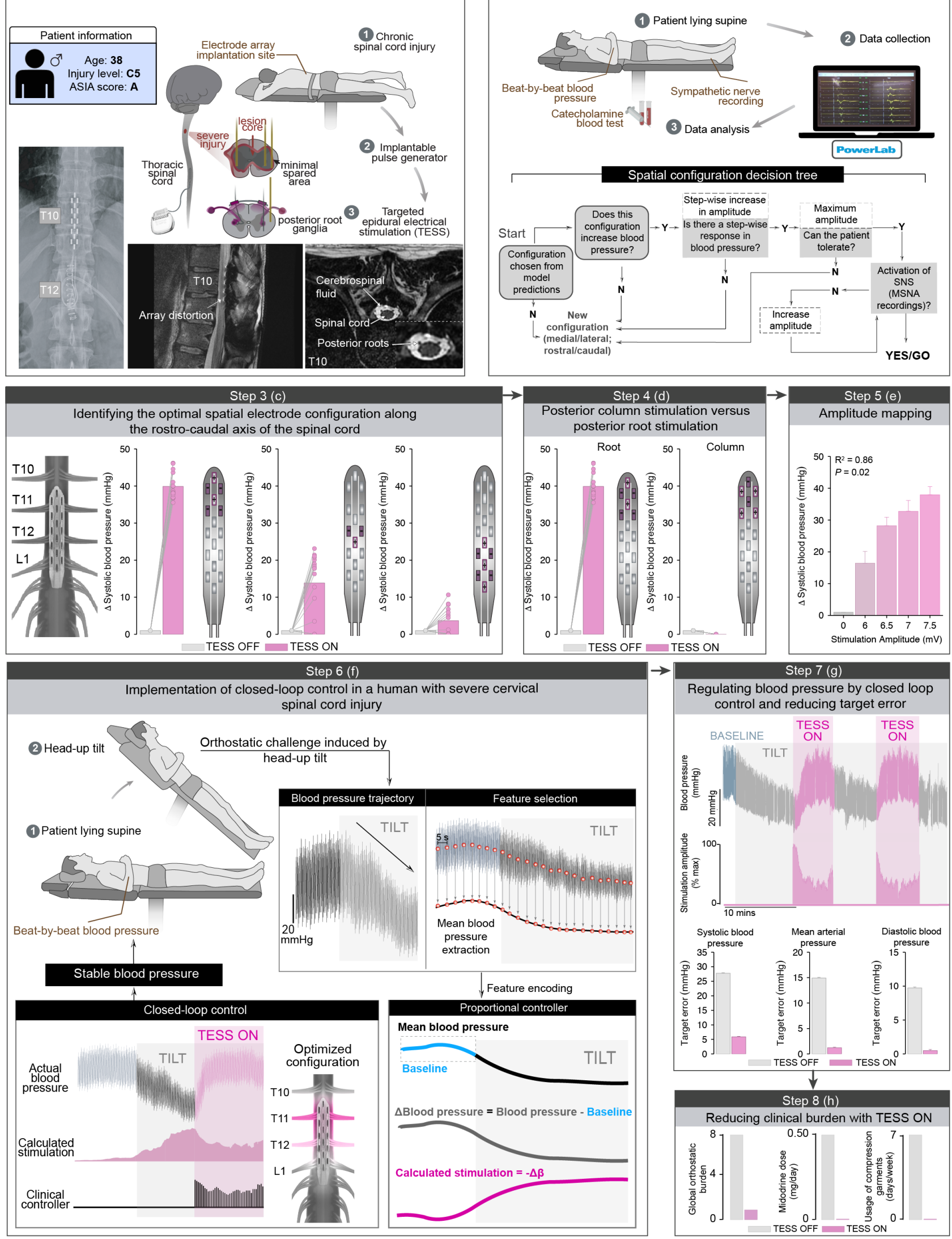


\section{Extended Data Figure 10. Targeted epidural electrical stimulation enables control of hemodynamics in a human with spinal cord injury.}

Step 1(a): We implanted a 5-6-5 Medtronic paddle array below the T10 and T11 vertebral bodies in a 38year-old patient with clinically-complete cervical spinal cord injury $(\mathrm{SCl})$ presenting with medically-refractory orthostatic hypotension. We confirmed the paddle array location with computerized tomography and magnetic resonance imaging.

Step 2(b): We recorded hemodynamics, sympathetic nerve activity and catecholamine levels in the patient without and with stimulation. We used personalized computational modelling and a genetic algorithm to predict the ideal spatial configurations to activate the lower thoracic spinal segments. We then used the illustrated decision-making process to determine whether to move forward with the use of a given configuration.

Step 3(c): We identified an optimal configuration, which recruited the lower thoracic spinal segments, increased blood pressure, normalized plasma norepinephrine levels, and increased muscle sympathetic nerve activity. We tested other configurations that are not optimally targeted to these roots and found the blood pressure responses were not robust.

Step 4(d): Activation of the posterior columns using medial electrodes did not lead to a pressor response.

Step 5(e): Using the optimal configuration, we found a stepwise increase in blood pressure as we increased the amplitude of stimulation ( $R^{2}=0.86 ; P=0.02$; linear regression).

Step 6(f): To test whether the concept of the neuroprosthetic baroreflex could be implemented in humans, we verticalized the patient to induce an orthostatic challenge while recording blood pressure. We performed feature extraction to obtain a rolling mean blood pressure, from which we calculated a continuous error (delta blood pressure). We then adjusted the weighted proportion to generate a calculated stimulation output, which was implemented using a clinician controller to modulate blood pressure in closed-loop.

Step 7(g): When our closed-loop system was activated, blood pressure was tightly regulated evidenced by stabilized blood pressure and mitigated target error.

Step 8(h): This patient now uses the stimulation daily and has been able to cease other treatments for orthostatic hypotension. Furthermore, the daily use of this therapy has reduced the self-reported burden of orthostatic hypotension for this patient, as assessed using clinical questionnaires.

Abbreviations: ASIA: American spinal injury association impairment scale; TESS: targeted epidural electrical spinal cord stimulation. 